\title{
Mobile Media and Social Media Addiction: A Survey
}

\author{
Niaz Ahmed \\ Saint Cloud State University, Minnesota, USA
}

\begin{abstract}
This study examined the smartphone and social media addiction among Generation Z. A survey was completed by a non-random sample of 415 students at State University of New York in Oneonta during the academic year 2017-2018. The results revealed that almost all Gen Zers (99\%) own a smartphone, and 98\% of them use their smartphones to connect to the Internet most of the time. More than half of Gen Zers spend 9 hours or more in a typical day using their smartphones, and nearly half of them spend 3-8 hours per day on their smartphones. With regard to the use of social media, more than half of Gen Zers accessed various social media several times a day, while nearly 1 in 5 admitted accessing social media every hour of the day. As for the amount of time devoted to social media, nearly one-third of the students admitted using social media for 7-10 hours per day, and slightly less than one-third of the students spent 5-6 hours per day on social media. With regard to Generation Z's perception of their addiction to smartphone and social media, 4 in 5 students admitted that they were spending an excessive amount of time on the Internet, and two-thirds believed that they were spending too much time on social media. Furthermore, 7 in 10 students admitted that they were addicted to the Internet, and nearly two-thirds believed they were addicted to social media. These findings may have significant implications for counseling and intervention for parents, educators, and policy makers.
\end{abstract}

Keywords: social media addiction, smartphone addiction, internet addiction

The advent of the smartphone and social media has significantly transformed our lives in terms of how we communicate and entertain ourselves. In recent years, smartphone and social media usage has grown exponentially among the general public and specifically within the Generation Z known as "Gen Z" or "Gen Zers" (Statista, 2015). The Generation Z is the demographic cohort born during the mid-1990s to mid-2000s (roughly 13-24 years of age today) and has followed the Millennials or Generation Y (Seemiller \& Grace, 2016; Wood, 2013). Generation Z is the first cohort to have smartphones, social media, and other technologies readily available at a young age. In terms of the size of Gen $\mathrm{Z}$ population, current estimate indicates that about one-third of world's 7.7 billion people are Gen Zers (Miller \& Lu, 2018).

While the advantages of the smartphone and social media are recognized, constant and excessive use may have negative consequences for the users such as addictive usage, increased stress, false sense of online connections, reduced face-to-face interactions, diminishing interpersonal skills, problematic family relations, and lower self-esteem (Hawi \& Samaha, 2016; Pew Research Center, 2016; Nazir \& Samaha, 2016; Bian \& Leung, 2014; Hong \& Chiu, 2012).

Niaz Ahmed, Dr., Professor Emeritus, Department of Mass Communication, Saint Cloud State University, Minnesota, USA. 
Although a significant amount of research has been done in the recent past about the use of smartphone and social media among Generation $\mathrm{X}$ and $\mathrm{Y}$, insufficient research exists about smartphone and social media addiction among Generation Z. Since the digital world is changing rapidly every year, it is important to track its usage and impacts on an ongoing basis for detecting any trends and correlations and for identifying significant implications for counseling and intervention for parents, educators, and policy makers. The present study attempts to contribute to the evolving knowledge by examining the implications of the addictive use of smartphone and social media among Generation Z. It also explores important variables that remained unexamined in past research.

\section{Literature Review}

Past studies have examined the extent and impact of smartphone and social media usage in the general public as well as among the Millennials. A survey (Lee, Sung, Lee, Lee, Cho, Park, \& Shin, 2016) of 490 adolescents found that $27 \%$ of them were in high risk of smartphone addiction, and showed significantly more behavioral and emotional problems, lower self-esteem, and poor quality of communication with their parents. A study by Nazir and Samaha in 2016 also revealed that addictive use of social media had a negative association with self-esteem. Wu, Cheung, Ku, and Hung (2013) also found a correlation between daily usage of smartphones and addictive symptoms towards social media. A study by Hong and Chiu (2012) revealed that students who scored high on smartphone addiction showed more social extraversion, anxiety, and lower self-esteem. An extensive review of literature by Kuss and Griffiths (2011) indicated that an excessive social media use may lead to symptoms traditionally associated with substance-related addictions.

A 2014 study of 414 university students (Bian \& Leung, 2014) identified five smartphone addiction symptoms: disregard of harmful consequences, preoccupation, inability to control craving, productivity loss, and feeling anxious and lost. The results showed that the higher one scored in loneliness, the higher the likelihood one would be addicted to smartphone. A study of 1,000 Belgians by Wang, Ho, Chan, and Tse (2015) found that compulsive users scored lower on emotional stability, conscientiousness, self-esteem, and higher on loneliness and depressive feelings.

Several studies have found that many smartphone users developed addictions as a consequence of using certain applications such as instant messaging, online gaming, and social networking through their mobile phones (Lopez-Fernandez, Kuss, Griffiths, \& Billieux, 2015; Billieux, Maurage, Lopez-Fernandez, Kuss, \& Griffiths, 2015; Kuss \& Griffiths, 2012).

In terms of sociodemographic differences, past research revealed that among those addicted to social media, females showed a higher prevalence of addictions and were more likely to experience anxiety and depression symptoms as a negative consequence of social media use (Bragazzi \& Del Puente, 2014; Caplan, 2003; Wang et al., 2015). In terms of age variation, past studies found that younger individuals were more likely to develop addictive behavior with social media (Song, LaRose, Eastin, \& Lin, 2004).

\section{Methodology}

A survey of college students was conducted to examine the smartphone and social media addiction among Generation Z during the academic year 2017-2018. The survey was administered online and was completed by 
a non-random sample of 415 students at State University of New York in Oneonta. This study poses the following research question:

What are the perceptions of Generation $\mathrm{Z}$ about their addiction to smartphone and social media? Are there any statistically significant differences in terms of gender and age?

\section{Results}

With a completion rate of 99\%, data were collected from a sample of 415 students at State University of New York in Oneonta. The response rate was predictably high due to the fact that the students received extra points for completing the survey as part of a class requirement. Approximately $56 \%$ of the respondents were females, and $43 \%$ were males. About $63 \%$ of the subjects were 18 -20 years of age, and $27 \%$ of them were 21-25. In terms of race, about $54 \%$ of the respondents were White and $46 \%$ were non-White (Hispanic, Black, Asian, Native American, Mixed). Approximately 48\% of the subjects were freshman and sophomores, and 42\% were juniors and seniors, while $10 \%$ were graduate students.

\section{How Does Generation Z Communicate?}

As shown in Table 1 and Figure 1, about 46\% of the students indicated that they used mediated interpersonal communication or digital communication (via smartphone, social media, email, texting, instant messaging, etc.) most often than face-to-face communication. Moreover, about 59\% of the students admitted that the use of social media had decreased their face-to-face communication (Figure 2).

Table 1

Comparison of Face-to-Face and Mediated Interpersonal Communication

\begin{tabular}{|c|c|c|}
\hline Types of communication & of resp & ondents \\
\hline I use face-to-face communication most of the time & $14.49 \%$ & 60 \\
\hline $\begin{array}{l}\text { I use mediated interpersonal communication most of the time (via smartphone, email, texting, online instant } \\
\text { messaging, social media, etc.). }\end{array}$ & $46.38 \%$ & 192 \\
\hline About one-third of my communication is mediated interpersonal communication & $27.54 \%$ & 114 \\
\hline About one-fourth of my communication is mediated interpersonal communication & $11.59 \%$ & 48 \\
\hline TOTAL & & 414 \\
\hline
\end{tabular}

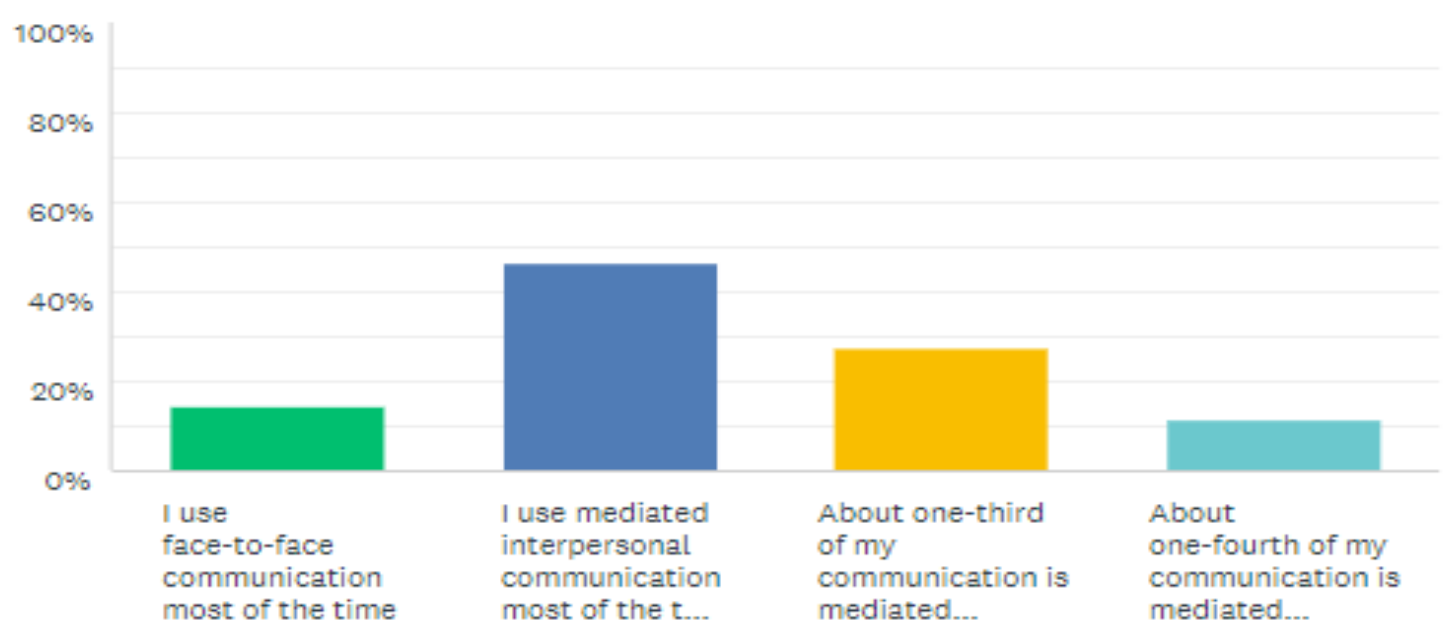

Figure 1. Comparison of face-to-face and mediated interpersonal communication. 


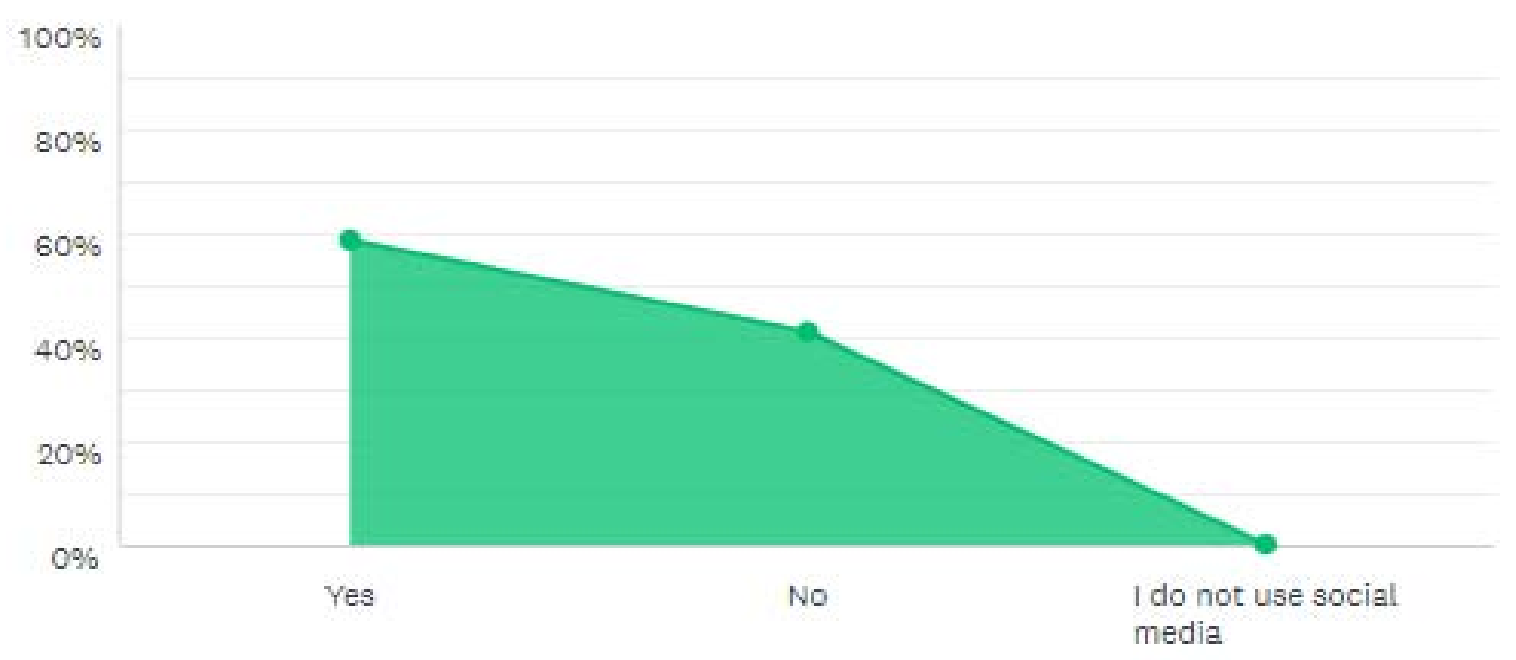

Figure 2. Have social media decreased face-to-face communication?

In terms of gender variation, the results did not show any statistically significant difference for most of the dimensions of this variable. Both males and females used mediated interpersonal communication more often than face-to-face communication. As shown in Table 2, significant difference was found for the response option "one-third of my communication is mediated interpersonal communication" whereby more female students (33\%) responded in affirmative than male students (20\%).

Table 2

Comparison of Face-to-Face and Mediated Interpersonal Communication by Gender

\begin{tabular}{|c|c|c|c|c|c|c|}
\hline Gende & & Frequency and perc & ent of respondents & & & \\
\hline & $\checkmark$ & $\begin{array}{l}\text { IUSE FACE-TO- } \\
\text { FACE } \\
\text { COMMUNICATION - } \\
\text { MOST OF THE } \\
\text { TIME }\end{array}$ & $\begin{array}{l}\text { I USE MEDIATED } \\
\text { INTERPPERSONAL } \\
\text { COMMUNICATION MOST OF } \\
\text { THE TIME (VIA } \\
\text { SMARTPHONE, EMAIL, } \\
\text { TEXTING, ONLINE INSTANT } \\
\text { MESSAGING, SOCIAL } \\
\text { MEDIA, ETC.). }\end{array}$ & $\begin{array}{l}\text { ABOUT ONE- } \\
\text { THIRD OF MY } \\
\text { COMMUNICATION } \\
\text { IS MEDIATED } \\
\text { INTERPERSONAL } \\
\text { COMMUNICATION }\end{array}$ & $\begin{array}{l}\text { ABOUT ONE- } \\
\text { FOURTH OF MY } \\
\text { COMMUNICATION } \\
\text { IS MEDIATED } \\
\text { INTERPERSONAL } \\
\text { COMMUNICATION }\end{array}$ & TOTAL \\
\hline$\checkmark$ & $\begin{array}{l}\text { Q90: Female } \\
\text { (A) }\end{array}$ & $\begin{array}{r}13.30 \% \\
31\end{array}$ & $\begin{array}{r}42.06 \% \\
98\end{array}$ & $\begin{array}{r}33.48 \% \\
78 \\
\mathrm{~B}\end{array}$ & $\begin{array}{r}11.16 \% \\
26\end{array}$ & $\begin{array}{r}56.42 \% \\
233\end{array}$ \\
\hline 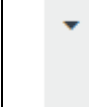 & $\begin{array}{l}\text { Q90: Male } \\
\text { (B) }\end{array}$ & $\begin{array}{r}16.11 \% \\
29\end{array}$ & $\begin{array}{r}51.67 \% \\
93\end{array}$ & $\begin{array}{r}20.00 \% \\
36 \\
\mathrm{~A}\end{array}$ & $\begin{array}{r}12.22 \% \\
22\end{array}$ & $\begin{array}{r}43.58 \% \\
180\end{array}$ \\
\hline$\checkmark$ & $\begin{array}{l}\text { Total } \\
\text { Respondents }\end{array}$ & 60 & 191 & 114 & 48 & 413 \\
\hline
\end{tabular}

Notes. ${ }^{*} \mathrm{p}=0.05,95 \%$ confidence.

As shown in Table 3, in terms of age variation, the results showed significant difference for most of the dimensions of this variable. More students in the age group 21-25 (64\%) indicated that they used mediated interpersonal communication compared to face-to-face communication most of the time. About $47 \%$ of the students in the age group 18-20 revealed that they used mediated interpersonal communication compared to face-to-face communication most of the time. 
Table 3

Comparison of Face-to-Face and Mediated Interpersonal Communication by Age

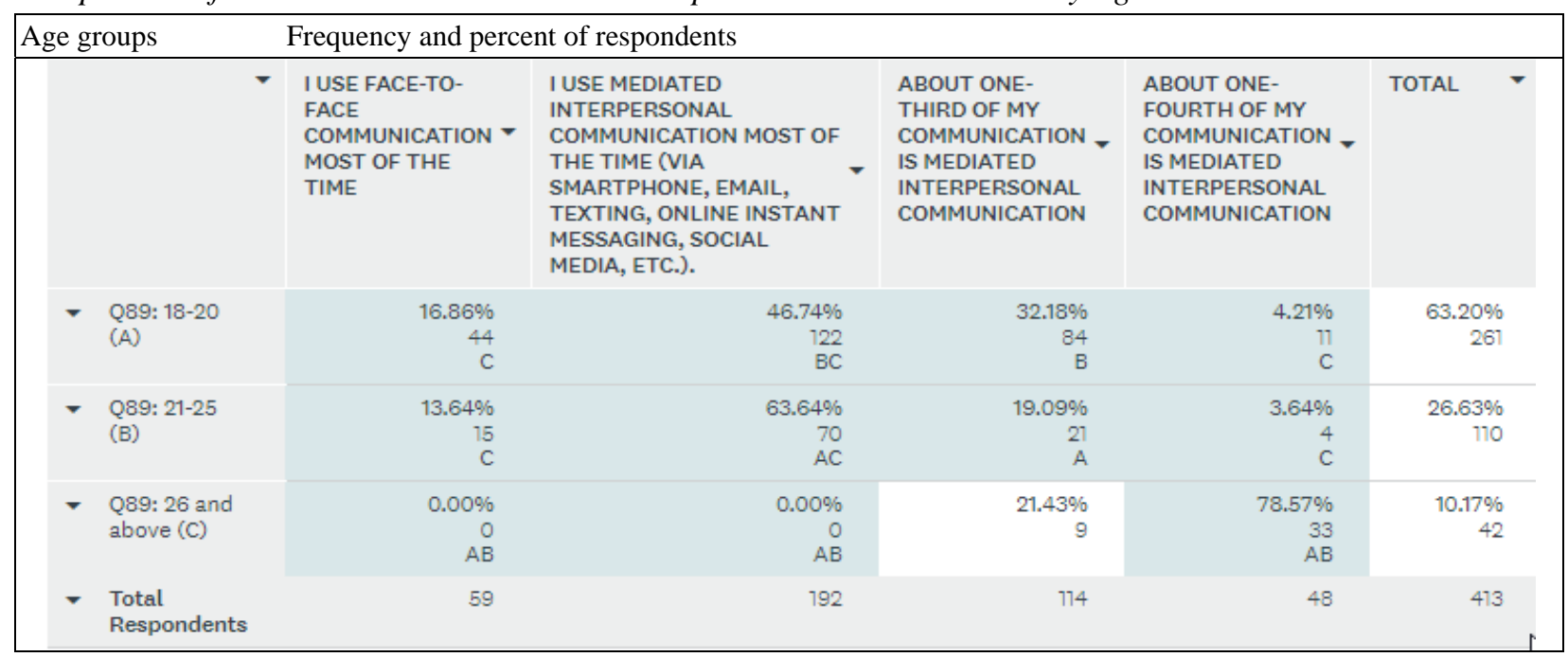

Notes. ${ }^{*} \mathrm{p}=0.05,95 \%$ confidence.

\section{How Does Generation Z Use Smartphones?}

According to survey results, $99 \%$ of the students had a functional smartphone, and about $98 \%$ of them used their smartphones to connect to the Internet most of the time (Table 4). Data did not show any significant difference in terms of gender for most of the dimensions of this variable. As shown in Table 5, only one dimension showed significant difference whereby more male students used game consoles to access the Internet compared to female students.

\section{Table 4}

Devices Used to Connect to the Internet Most of the Time

\begin{tabular}{|c|c|c|c|}
\hline Types of devices & & \multicolumn{2}{|c|}{ Frequency and percent of respondents } \\
\hline ANSWER CHOICES & $\boldsymbol{v}$ & RESPONSES & $\boldsymbol{v}$ \\
\hline Desktop computer & & $4.58 \%$ & 19 \\
\hline Laptop computer & & $38.07 \%$ & 158 \\
\hline Computer tablet & & $5.30 \%$ & 22 \\
\hline Smartphone & & $97.59 \%$ & 405 \\
\hline - Digital assistant (EDA or PDA) & & $0.96 \%$ & 4 \\
\hline Game console & & $5.78 \%$ & 24 \\
\hline Smart TV & & $1.69 \%$ & 7 \\
\hline Other (please specify) & Responses & $0.72 \%$ & 3 \\
\hline Total Respondents: 415 & & & \\
\hline
\end{tabular}


Table 5

Devices Used to Connect to the Internet Most of the Time by Gender

\begin{tabular}{|c|c|c|c|c|c|c|c|c|c|}
\hline \multirow[t]{2}{*}{ Gender } & \multicolumn{9}{|c|}{ Frequency and percent of respondents } \\
\hline & $\begin{array}{l}\text { DESKTOP } \\
\text { COMPUTER }\end{array}$ & $\begin{array}{l}\text { LAPTOP } \\
\text { COMPUTER }\end{array}$ & $\begin{array}{l}\text { COMPUTER } \\
\text { TABLET }\end{array}$ & SMARTPHONE & $\begin{array}{l}\text { DIGITAL } \\
\text { ASSISTANT } \\
\text { (EDA OR } \\
\text { PDA) }\end{array}$ & $\begin{array}{l}\text { GAME } \\
\text { CONSOLE }\end{array}$ & $\begin{array}{l}\text { SMART, } \\
\text { TV }\end{array}$ & $\begin{array}{l}\text { OTHER } \\
\text { (PLEASE } \\
\text { SPECIFY) }\end{array}$ & TOTAL $\boldsymbol{V}$ \\
\hline $\begin{array}{l}\text { Q90: Female } \\
\text { (A) }\end{array}$ & $\begin{array}{r}3.02 \% \\
11\end{array}$ & $\begin{array}{r}26.65 \% \\
97\end{array}$ & $\begin{array}{r}3.85 \% \\
14\end{array}$ & $\begin{array}{r}63.74 \% \\
232\end{array}$ & $\begin{array}{r}0.55 \% \\
2\end{array}$ & $\begin{array}{r}1.10 \% \\
4 \\
B\end{array}$ & $\begin{array}{r}0.82 \% \\
3\end{array}$ & $\begin{array}{r}0.27 \% \\
1 \\
\text { Responses }\end{array}$ & $\begin{array}{r}87.92 \% \\
364\end{array}$ \\
\hline $\begin{array}{l}\text { Q90: Male } \\
\text { (B) }\end{array}$ & $\begin{array}{r}2.55 \% \\
7\end{array}$ & $\begin{array}{r}22.18 \% \\
61\end{array}$ & $\begin{array}{r}2.55 \% \\
7\end{array}$ & $\begin{array}{r}62.55 \% \\
172\end{array}$ & $\begin{array}{r}0.73 \% \\
2\end{array}$ & $\begin{array}{r}7.27 \% \\
20 \\
\mathrm{~A}\end{array}$ & $\begin{array}{r}1.45 \% \\
4\end{array}$ & $\begin{array}{r}0.73 \% \\
2 \\
\text { Responses }\end{array}$ & $\begin{array}{r}66.43 \% \\
275\end{array}$ \\
\hline $\begin{array}{l}\text { Total } \\
\text { Respondents }\end{array}$ & 18 & 158 & 21 & 404 & 4 & 24 & 7 & 3 & 414 \\
\hline
\end{tabular}

Notes. ${ }^{*} \mathrm{p}=0.05,95 \%$ confidence.

As shown in Table 6, in terms of age variation, data revealed significant difference for two dimensions. More students in the older age groups (21-25 and 26+) used smartphones to access the Internet (66\% and 100\% respectively) most of the time than students in the 18-20 age group (59\%). However, more students in the younger age groups (18-20 and 21-25) used laptops to access the Internet most of the time (29\% and $20 \%$ respectively) compared to older students (26+) who did not use laptops to access the Internet.

Table 6

Devices Used to Connect to the Internet Most of the Time by Age

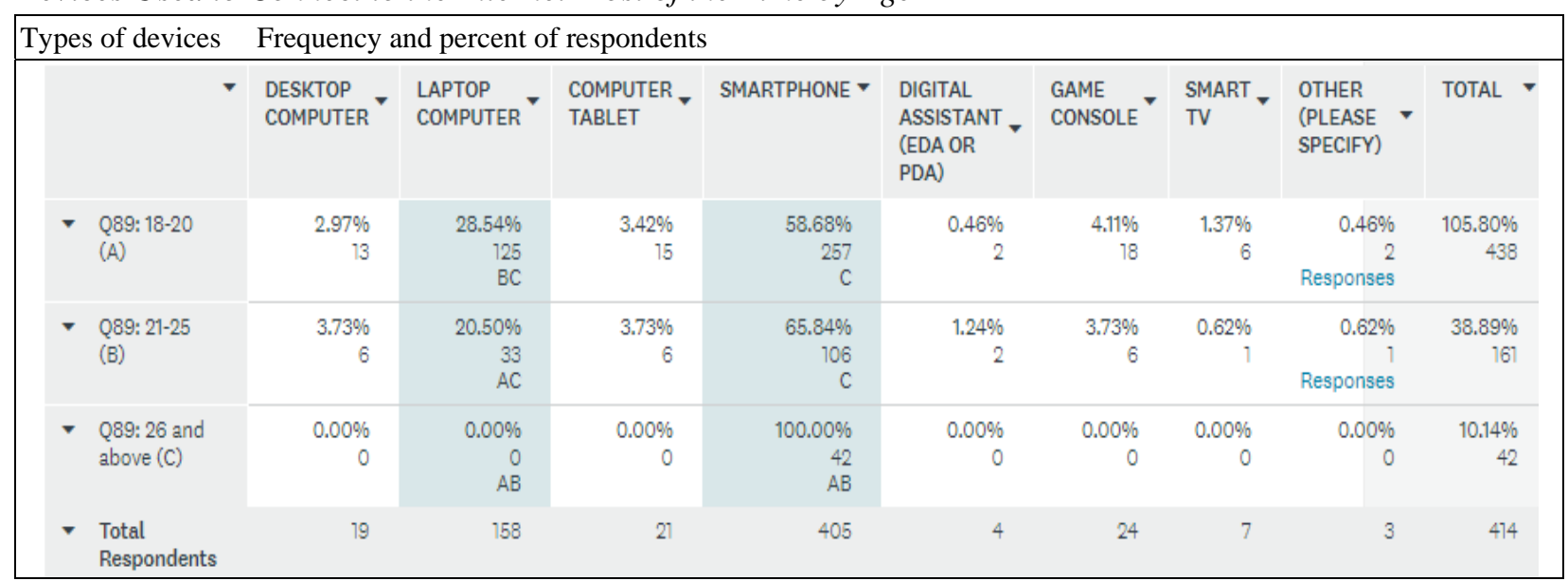

Notes. ${ }^{*} \mathrm{p}=0.05,95 \%$ confidence.

As shown in Table 7, approximately 51\% of the students spent nine hours or more in a typical day using their smartphones, and about 48\% spent 3-8 hours per day on their smartphones. Data did not show any significant difference in terms of gender or age with regard to the amount of time spent on smartphones (Tables 8 and 9). 
Table 7

Amount of Time Spent in a Typical Day Using the Smartphone

\begin{tabular}{|lrc|}
\hline Amount of time & Frequency and percent of respondents \\
\hline 3-8 hours & $48.43 \%$ & 201 \\
\hline 9 hours or more & $51.33 \%$ & 213 \\
\hline I do not use a smartphone & $0.24 \%$ & 1 \\
TOTAL & & 415 \\
\hline
\end{tabular}

Table 8

Amount of Time Spent in a Typical Day Using the Smartphone by Gender

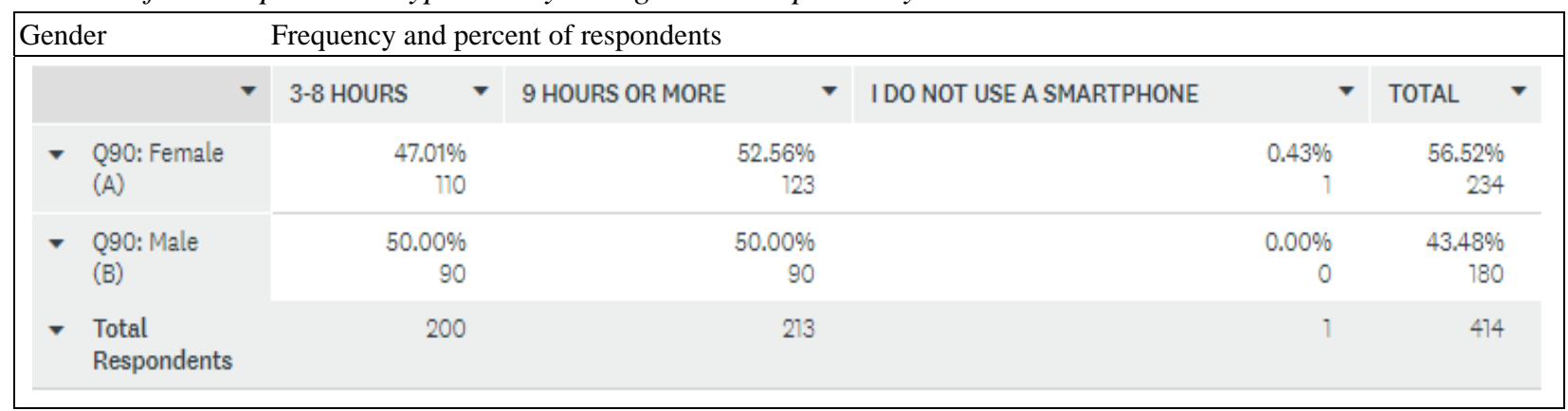

Table 9

Amount of Time Spent in a Typical Day Using the Smartphone by Age

\begin{tabular}{|c|c|c|c|c|c|}
\hline Age groups & \multicolumn{5}{|c|}{ Frequency and percent of respondents } \\
\hline$\vec{v}$ & 3-8 HOURS & 9 HOURS OR MORE & I DO NOT USE A SMARTPHONE & $\boldsymbol{v}$ & TOTAL \\
\hline $\begin{array}{l}\text { Q89: } 18-20 \\
\text { (A) }\end{array}$ & $\begin{array}{r}47.33 \% \\
124\end{array}$ & $\begin{array}{r}52.67 \% \\
138\end{array}$ & & $\begin{array}{r}0.00 \% \\
0\end{array}$ & $\begin{array}{r}63.29 \% \\
262\end{array}$ \\
\hline $\begin{array}{l}\text { Q89:21-25 } \\
\text { (B) }\end{array}$ & $\begin{array}{r}32.73 \% \\
36\end{array}$ & $\begin{array}{r}67.27 \% \\
74\end{array}$ & & $\begin{array}{r}0.00 \% \\
0\end{array}$ & $\begin{array}{r}26.57 \% \\
110\end{array}$ \\
\hline $\begin{array}{l}\text { Q89: } 26 \text { and } \\
\text { above (C) }\end{array}$ & $\begin{array}{r}97.62 \% \\
41\end{array}$ & $\begin{array}{r}2.38 \% \\
1\end{array}$ & & $\begin{array}{r}0.00 \% \\
0\end{array}$ & $\begin{array}{r}10.14 \% \\
42\end{array}$ \\
\hline $\begin{array}{l}\text { Total } \\
\text { Respondents }\end{array}$ & 201 & 213 & & 0 & 414 \\
\hline
\end{tabular}

With regard to the nature of smartphone usage, Table 10 shows that about $95 \%$ of the students used their smartphones to make or receive phone calls, $96 \%$ used it to send or receive text messages, and $92 \%$ used it to send or receive emails. About $80 \%$ used their smartphones to take, post, and view photos and videos on social media, and to check the news online. Furthermore, about $66 \%$ of the students used their smartphones to watch movies online, $97 \%$ used it to play music, $9 \%$ used it to watch TV online, and $75 \%$ used it for playing games. In addition, about $40 \%$ used it for online purchasing, $29 \%$ used it for accessing dating sites, and $12 \%$ used it to search or apply for jobs. One notable finding revealed that $67 \%$ of the students used their smartphones for doing research for class assignments. 
Table 10

Type of Activities Performed on Smartphones

\begin{tabular}{|c|c|c|}
\hline Types of activities & Frequency and per & ondents \\
\hline Make or receive phone calls & $95.18 \%$ & 395 \\
\hline Send or receive texts or instant messages & $96.39 \%$ & 400 \\
\hline - Send or receive emails & $92.05 \%$ & 382 \\
\hline Record videos & $79.76 \%$ & 331 \\
\hline Send or post videos & $82.17 \%$ & 341 \\
\hline Watch videos & $89.64 \%$ & 372 \\
\hline Watch movies & $65.54 \%$ & 272 \\
\hline Take photos & $91.33 \%$ & 379 \\
\hline Send or post photos & $88.67 \%$ & 368 \\
\hline Play music & $96.63 \%$ & 401 \\
\hline - Play games & $75.18 \%$ & 312 \\
\hline Play podcasts & $12.29 \%$ & 51 \\
\hline Surf the internet & $88.92 \%$ & 369 \\
\hline Purchase products or services & $40.24 \%$ & 167 \\
\hline - Use dating sites & $29.16 \%$ & 121 \\
\hline Check the news & $79.52 \%$ & 330 \\
\hline - Use social media such as Facebook, Twitter, and Snapchat & $93.01 \%$ & 386 \\
\hline Research for class assignments & $67.23 \%$ & 279 \\
\hline Search or apply for jobs & $11.81 \%$ & 49 \\
\hline Watch TV & $9.40 \%$ & 39 \\
\hline Taking memos, notes, etc. & $13.98 \%$ & 58 \\
\hline Other (please specify) & Responses & 4 \\
\hline Total Respondents: 415 & & \\
\hline
\end{tabular}

The data did not show any significant difference in terms of gender for most of the dimensions of this variable. As shown in Table 11, only three of the above dimensions showed significant difference whereby more male students used smartphones (18\%) to play podcast compared to female students (8\%), and 34\% males used their smartphones to access dating sites compared to $25 \%$ females. However, more female students (15\%) used their smartphones to apply for jobs online compared to males (8\%). 
Table 11

Types of Activities Performed on Smartphones by Gender

\begin{tabular}{|c|c|c|c|c|c|c|c|c|c|c|c|c|c|}
\hline \multicolumn{14}{|c|}{ Types of activities Frequency and percent of respondents } \\
\hline & \begin{tabular}{|l|l|} 
MAKE & OR \\
RECE \\
PHON \\
CALL
\end{tabular} & $\begin{array}{l}\text { E } \\
\text { IVE - } \\
\text { NE } \\
\text { S }\end{array}$ & $\begin{array}{l}\text { SEND C } \\
\text { RECEIV } \\
\text { TEXTS } \\
\text { INSTAN } \\
\text { MESSA }\end{array}$ & $\begin{array}{l}\text { R } \\
\text { E } \\
\text { OR - } \\
\text { GT } \\
\text { GES }\end{array}$ & $\begin{array}{l}\text { SEND } \\
\text { OR } \\
\text { RECEIVE } \\
\text { EMAILS }\end{array}$ & $\begin{array}{l}\text { RECORD } \\
\text { VIDEOS }\end{array}$ & $\begin{array}{l}\text { END } \\
\text { R } \\
\text { OST } \\
\text { IDEOS }\end{array}$ & $\begin{array}{l}\text { WATCH } \\
\text { VIDEOS }\end{array}$ & $\begin{array}{l}\text { WATCH } \\
\text { MOVIES }\end{array}$ & $\begin{array}{l}\text { TAKE } \\
\text { РHOTOS }\end{array}$ & $\begin{array}{l}\text { SEND } \\
\text { OR } \\
\text { POST } \\
\text { PHOTOS }\end{array}$ & ${ }_{\text {MUSIC }}^{\text {PLAY }}$ & $\begin{array}{l}\text { PLAY } \\
\text { GAMES }\end{array}$ \\
\hline \multicolumn{2}{|c|}{$\begin{array}{l}\text { Q90: Female } \\
\text { (A) }\end{array}$} & $\begin{array}{l}73 \% \\
224\end{array}$ & \multicolumn{2}{|c|}{$\begin{array}{r}97.86 \% \\
229\end{array}$} & $\begin{array}{r}92.74 \% \\
217\end{array}$ & $\begin{array}{r}82.05 \% \\
192\end{array}$ & $\begin{array}{r}3.76 \% \\
196\end{array}$ & $\begin{array}{r}92.31 \% \\
216\end{array}$ & $\begin{array}{r}65.81 \% \\
154\end{array}$ & $\begin{array}{r}95.73 \% \\
224\end{array}$ & $\begin{array}{r}91.45 \% \\
214\end{array}$ & $\begin{array}{r}98.72 \% \\
231\end{array}$ & $\begin{array}{r}73.93 \% \\
173\end{array}$ \\
\hline \multicolumn{2}{|c|}{$\begin{array}{l}\text { Q90: Male } \\
\text { (B) }\end{array}$} & $\begin{array}{l}14 \% \\
170\end{array}$ & \multicolumn{2}{|c|}{$\begin{array}{r}94.44 \% \\
170\end{array}$} & $\begin{array}{r}91.11 \% \\
164\end{array}$ & $\begin{array}{r}76.67 \% \\
138\end{array}$ & $\begin{array}{r}0.00 \% \\
144\end{array}$ & $\begin{array}{r}86.11 \% \\
155\end{array}$ & $\begin{array}{r}65.56 \% \\
118\end{array}$ & $\begin{array}{r}85.56 \% \\
154\end{array}$ & $\begin{array}{r}85.00 \% \\
153\end{array}$ & $\begin{array}{r}93.89 \% \\
169\end{array}$ & $\begin{array}{r}77.22 \% \\
139\end{array}$ \\
\hline \multicolumn{2}{|c|}{$\begin{array}{l}\text { Total } \\
\text { Respondents }\end{array}$} & 394 & & 399 & 381 & 330 & 340 & 371 & 272 & 378 & 367 & 400 & 312 \\
\hline $\begin{array}{l}\text { PLAY } \\
\text { PODCASTS }\end{array}$ & $\begin{array}{l}\text { SURF THE } \\
\text { INTERNET }\end{array}$ & $\begin{array}{l}\text { PURC } \\
\text { PROD } \\
\text { OR } \\
\text { SERV }\end{array}$ & $\begin{array}{l}\text { CHASE } \\
\text { DUCTS } \\
\text { ICES }\end{array}$ & $\begin{array}{l}\text { USE } \\
\text { DATING } \\
\text { SITES }\end{array}$ & $\begin{array}{l}\text { CHECK } \\
\text { THE } \\
\text { NEWS }\end{array}$ & $\begin{array}{l}\text { USE } \\
\text { SOCIAL } \\
\text { MEDIA } \\
\text { SUCHAS } \\
\text { FACEBOOK, } \\
\text { TWITTER, } \\
\text { AND } \\
\text { SNAPCHAT }\end{array}$ & \multicolumn{2}{|c|}{$\begin{array}{l}\text { RESEARCH } \\
\text { FOR CLASS } \\
\text { ASSIGNMENTS }\end{array}$} & $\begin{array}{l}\text { SEARCH } \\
\text { OR } \\
\text { APPLY - } \\
\text { FOR } \\
\text { JOBS }\end{array}$ & $\begin{array}{l}\text { WATCH } \\
\text { TV }\end{array}$ & $\begin{array}{l}\text { TAKING } \\
\text { MEMOS, } \\
\text { NOTES, } \\
\text { ETC. }\end{array}$ & $\begin{array}{l}\text { OTHER } \\
\text { (PLEASE } \\
\text { SPECIFY) }\end{array}$ & TOTAL \\
\hline $\begin{array}{r}8.12 \% \\
19 \\
B\end{array}$ & $\begin{array}{r}89.74 \% \\
210\end{array}$ & & $\begin{array}{r}41.45 \% \\
97\end{array}$ & $\begin{array}{r}24.79 \% \\
58 \\
B\end{array}$ & $\begin{array}{r}79.06 \% \\
185\end{array}$ & $\begin{array}{r}95.30 \% \\
223\end{array}$ & & $\begin{array}{r}71.79 \% \\
168\end{array}$ & $\begin{array}{r}14.96 \% \\
35 \\
B\end{array}$ & $\begin{array}{r}10.68 \% \\
25\end{array}$ & $\begin{array}{r}16.67 \% \\
39\end{array}$ & $\begin{array}{r}1.28 \% \\
3 \\
\text { Responses }\end{array}$ & $\begin{array}{r}804.83 \% \\
3,332\end{array}$ \\
\hline $\begin{array}{r}17.78 \% \\
32 \\
\mathrm{~A}\end{array}$ & $\begin{array}{r}87.78 \% \\
158\end{array}$ & & $\begin{array}{r}8.33 \% \\
69\end{array}$ & $\begin{array}{r}34.44 \% \\
62 \\
\mathrm{~A}\end{array}$ & $\begin{array}{r}80.00 \% \\
144\end{array}$ & $\begin{array}{r}90.00 \% \\
162\end{array}$ & & $\begin{array}{r}61.67 \% \\
\text { ווו }\end{array}$ & $\begin{array}{r}7.78 \% \\
14 \\
\mathrm{~A}\end{array}$ & $\begin{array}{r}7.78 \% \\
14\end{array}$ & $\begin{array}{r}10.00 \% \\
18\end{array}$ & $\begin{array}{r}0.56 \% \\
1 \\
\text { Responses }\end{array}$ & $\begin{array}{r}593.96 \% \\
2,459\end{array}$ \\
\hline 51 & 368 & & 166 & 120 & 329 & 385 & & 279 & 49 & 39 & 57 & 4 & 474 \\
\hline
\end{tabular}

Notes. ${ }^{*} \mathrm{p}=0.05,95 \%$ confidence.

In terms of age variation, data revealed significant differences for five dimensions as shown in Table 12. More students in the older age groups (21-25 and 26+) used smartphones to check the news online (84\% and $100 \%$ respectively) than students in the 18-20 age group (75\%). However, more students in the younger age groups (18-20 and 21-25) used their smartphones to apply for jobs online (15\% and $8 \%$ respectively) than older students (26+) who did not use smartphones for this purpose at all. Furthermore, more students in the younger age groups (18-20 and 21-25) used their smartphones to watch TV online (11\% and 9\% respectively) than older students (26+) who did not use smartphones for this purpose. 
Table 12

Types of Activities Performed on Smartphones by Age

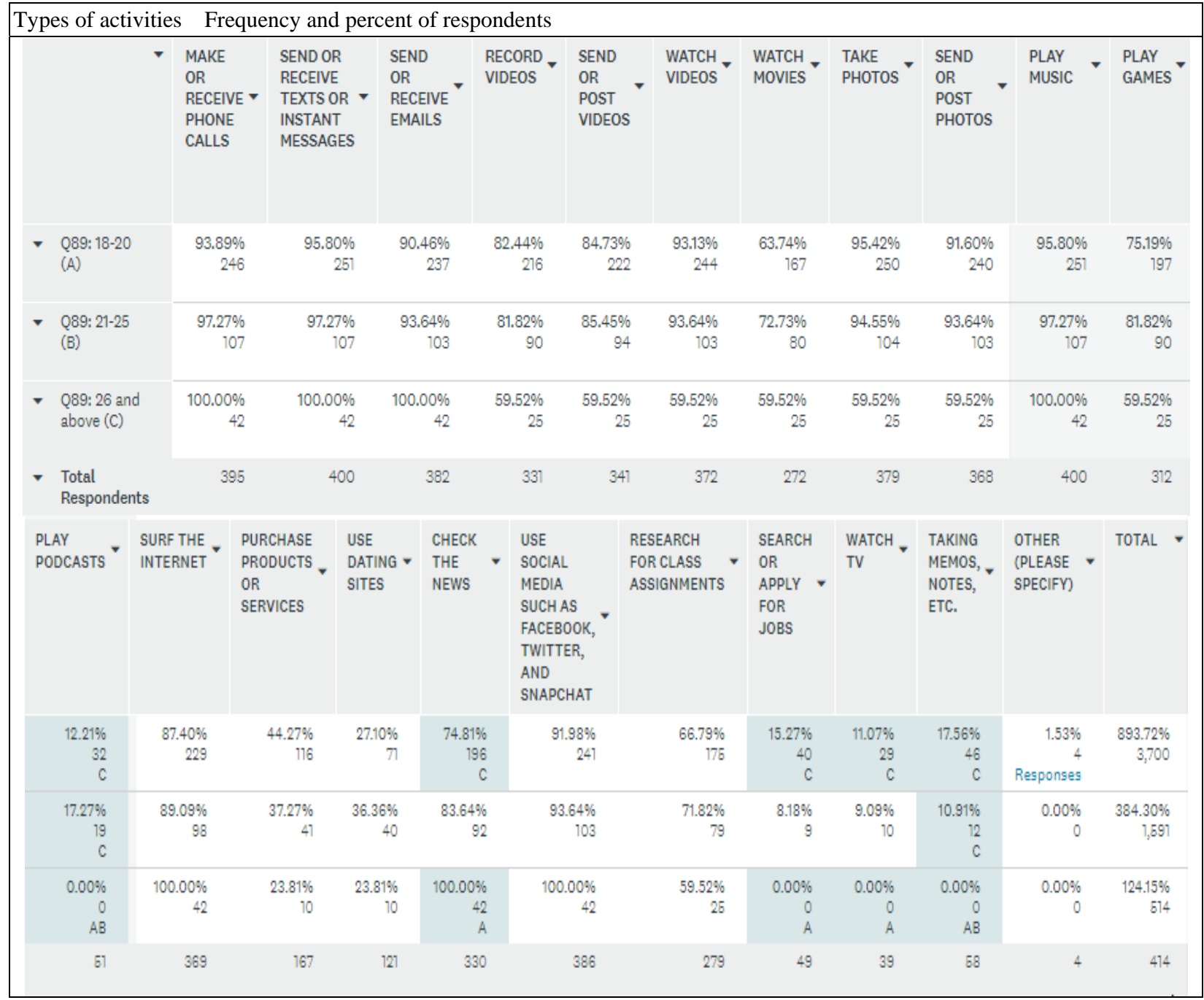

Notes. ${ }^{*} \mathrm{p}=0.05,95 \%$ confidence.

\section{How Does Generation Z Use Social Media?}

The results revealed that Snapchat, Instagram, Facebook, and Twitter are the most widely used social media among Generation $\mathrm{Z}$ with majority of the respondents having multiple social media accounts. As shown in Table 13, 93\% of the students had an account on Facebook, 95\% had an account on Snapchat, 88\% had an account on Twitter, and 58\% had an account on Instagram. In addition, about $41 \%$ used YouTube while 15\% used Google Plus. 
I do not have an account o...

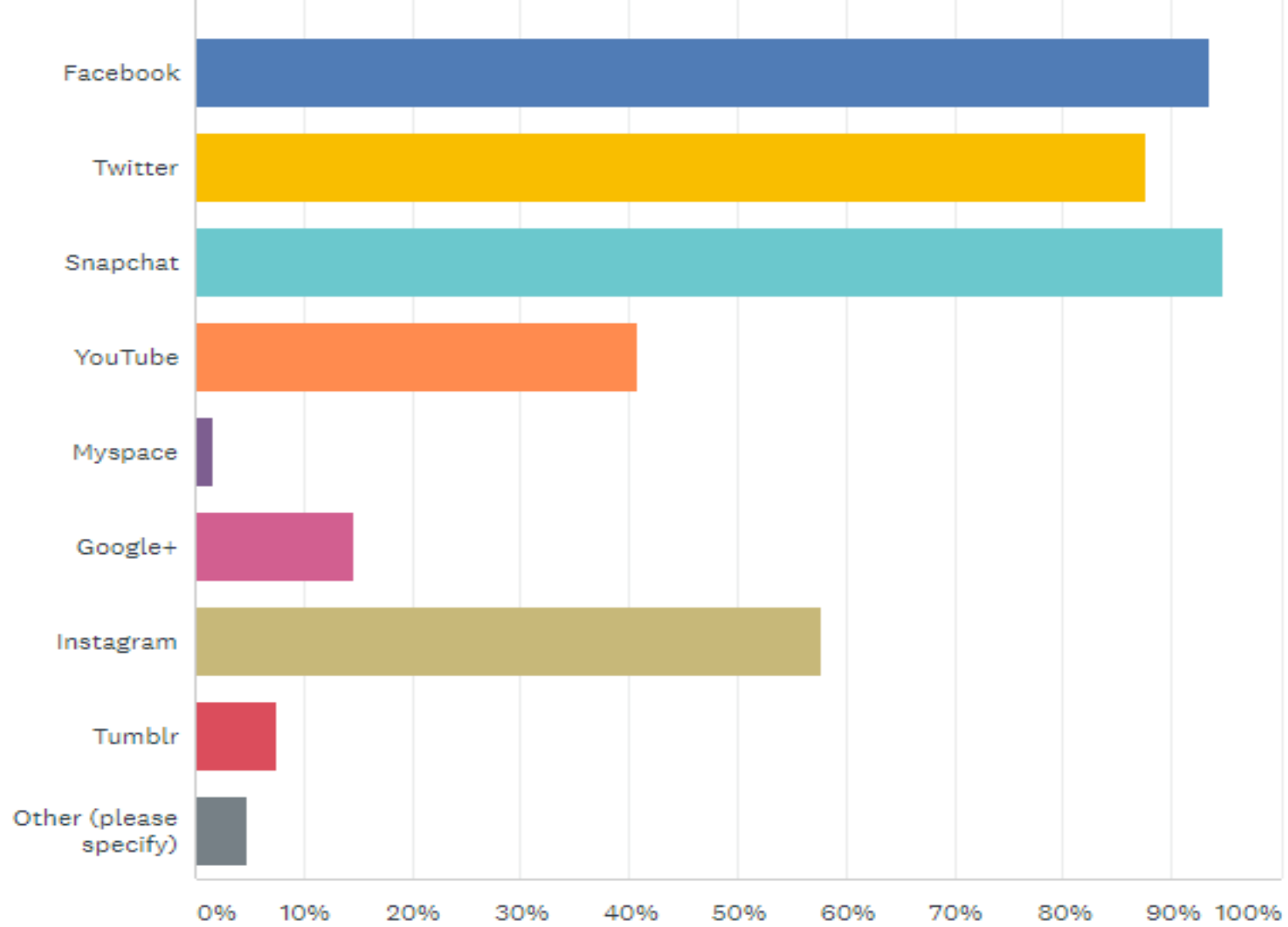

Figure 3. Most frequently used social media.

Table 13

Most Frequently Used Social Media

\begin{tabular}{|c|c|c|c|}
\hline Types of social media & \multicolumn{3}{|c|}{ Frequency and percent of respondents } \\
\hline I do not have an account on any social media & & $0.24 \%$ & 1 \\
\hline Facebook & & $93.49 \%$ & 388 \\
\hline Twitter & & $87.71 \%$ & 364 \\
\hline Snapchat & & $94.70 \%$ & 393 \\
\hline YouTube & & $40.72 \%$ & 169 \\
\hline Myspace & & $1.69 \%$ & 7 \\
\hline Google+ & & $14.70 \%$ & 61 \\
\hline Instagram & & $57.83 \%$ & 240 \\
\hline Tumblr & & $7.47 \%$ & 31 \\
\hline Other (please specify) & Responses & $4.82 \%$ & 20 \\
\hline Total Respondents: 415 & & & \\
\hline
\end{tabular}

In terms of frequency of usage, $52 \%$ of the respondents indicated that they accessed various social media several times a day, while $18 \%$ admitted accessing social media every hour of the day (Table 14). 
Table 14

Frequency of Social Media Usage

\begin{tabular}{|lcc|}
\hline How often social media are used & Frequency and percent of respondents \\
\hline Never & $0.96 \%$ & 4 \\
\hline A few times a month & $0.48 \%$ & 2 \\
\hline A few times a week & $4.34 \%$ & 18 \\
\hline About once a day & $24.82 \%$ & 103 \\
\hline Several times a day & $51.57 \%$ & 214 \\
\hline TOTAL & $17.83 \%$ & 74 \\
\hline
\end{tabular}

As shown in Table 15, in terms of gender variation, significant difference was found for the response option "about once a day" whereby more male students (33\%) responded in affirmative than female students (19\%). Conversely, for the response option "every hour of the day" more female students (25\%) responded in affirmative than male students (9\%).

Table 15

Frequency of Social Media Usage by Gender

\begin{tabular}{|c|c|c|c|c|c|c|c|}
\hline \multirow[t]{2}{*}{ Gender } & \multicolumn{7}{|c|}{ Frequency and percent of respondents } \\
\hline & NEVER $\boldsymbol{v}$ & $\begin{array}{l}\text { A FEW } \\
\text { TIMES A } \\
\text { MONTH }\end{array}$ & $\begin{array}{l}\text { A FEW } \\
\text { TIMES A } \\
\text { WEEK }\end{array}$ & $\begin{array}{l}\text { ABOUT } \\
\text { ONCE A } \\
\text { DAY }\end{array}$ & $\begin{array}{l}\text { SEVERAL } \\
\text { TIMES A } \\
\text { DAY }\end{array}$ & $\begin{array}{l}\text { EVERY HOUR } \\
\text { OF THE DAY }\end{array}$ & TOTAL \\
\hline $\begin{array}{l}\text { Q90: Female } \\
\text { (A) }\end{array}$ & $\begin{array}{r}0.85 \% \\
2\end{array}$ & $\begin{array}{r}0.85 \% \\
2\end{array}$ & $\begin{array}{r}2.99 \% \\
7\end{array}$ & $\begin{array}{r}18.80 \% \\
44 \\
B\end{array}$ & $\begin{array}{r}51.71 \% \\
121\end{array}$ & $\begin{array}{r}24.79 \% \\
58 \\
B\end{array}$ & $\begin{array}{r}56.52 \% \\
234\end{array}$ \\
\hline $\begin{array}{l}\text { Q90: Male } \\
\text { (B) }\end{array}$ & $\begin{array}{r}1.11 \% \\
2\end{array}$ & $\begin{array}{r}0.00 \% \\
0\end{array}$ & $\begin{array}{r}6.11 \% \\
11\end{array}$ & $\begin{array}{r}32.78 \% \\
59 \\
\mathrm{~A}\end{array}$ & $\begin{array}{r}51.11 \% \\
92\end{array}$ & $\begin{array}{r}8.89 \% \\
16 \\
\mathrm{~A}\end{array}$ & $\begin{array}{r}43.48 \% \\
180\end{array}$ \\
\hline $\begin{array}{l}\text { Total } \\
\text { Respondents }\end{array}$ & 4 & 2 & 18 & 103 & 213 & 74 & 414 \\
\hline
\end{tabular}

Notes. ${ }^{*} \mathrm{p}=0.05,95 \%$ confidence.

In terms of age variation, the data showed significant differences for most of the dimensions. As shown in Table 16, the younger students accessed the social media more often than the older students. 
Table 16

Frequency of Social Media Usage by Age

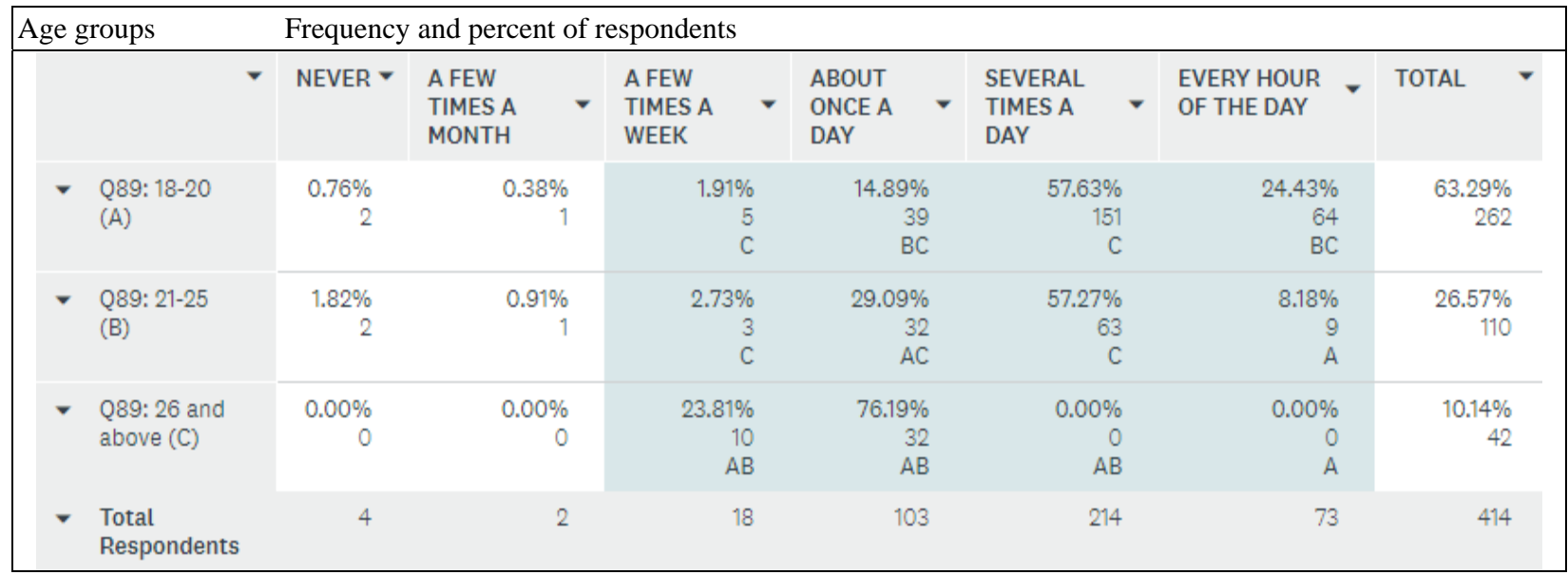

Notes. ${ }^{*} \mathrm{p}=0.05,95 \%$ confidence.

With regard to the amount of time devoted to social media, approximately $32 \%$ of the students admitted using social media for 7-10 hours per day, 30\% spent 5-6 hours per day, and 29\% spent 1-2 hours a day (Table 17).

Table 17

Amount of Time Spent on Social Media

\begin{tabular}{|lcr|}
\hline Amount of time & Frequency and percent of respondents \\
\hline 0 hours & $1.20 \%$ & 5 \\
\hline 1-2 hours & $29.40 \%$ & 122 \\
\hline$\quad 5-6$ hours & $29.88 \%$ & 124 \\
\hline$\quad 7-10$ hours & $31.57 \%$ & 131 \\
\hline 11 hours or more & $7.95 \%$ & 33 \\
TOTAL & & 415 \\
\hline
\end{tabular}

In terms of gender variation, the results showed significant difference for two dimensions. More females (14\%) used social media for 11 hours or more per day than males (0.56\%). Conversely, more male students used (37\%) social media for 1-2 hours per day than female (24\%) students (Table 18).

Table 18

Amount of Time Spent on Social Media by Gender

\begin{tabular}{|c|c|c|c|c|c|c|}
\hline \multirow[t]{2}{*}{ Gender } & \multicolumn{6}{|c|}{ Frequency and percent of respondents } \\
\hline & O HOURS - & 1-2 HOURS & 5-6 HOURS - & 7-10 HOURS - & 11 HOURS OR MORE & TOTAL \\
\hline $\begin{array}{l}\text { Q90: Female } \\
\text { (A) }\end{array}$ & $\begin{array}{r}0.43 \% \\
1\end{array}$ & $\begin{array}{r}23.93 \% \\
56 \\
\mathrm{~B}\end{array}$ & $\begin{array}{r}31.62 \% \\
74\end{array}$ & $\begin{array}{r}30.34 \% \\
71\end{array}$ & $\begin{array}{r}13.68 \% \\
32 \\
\mathrm{~B}\end{array}$ & $\begin{array}{r}56.52 \% \\
234\end{array}$ \\
\hline $\begin{array}{l}\text { Q90: Male } \\
\text { (B) }\end{array}$ & $\begin{array}{r}2.22 \% \\
4\end{array}$ & $\begin{array}{r}36.67 \% \\
66 \\
\mathrm{~A}\end{array}$ & $\begin{array}{r}27.78 \% \\
50\end{array}$ & $\begin{array}{r}32.78 \% \\
59\end{array}$ & $\begin{array}{r}0.56 \% \\
1 \\
\text { A }\end{array}$ & $\begin{array}{r}43.48 \% \\
180\end{array}$ \\
\hline $\begin{array}{l}\text { Total } \\
\text { Respondents }\end{array}$ & 5 & 122 & 124 & 130 & 33 & 474 \\
\hline
\end{tabular}

Notes. ${ }^{*} \mathrm{p}=0.05,95 \%$ confidence. 
In terms of age variation, the data showed significant difference for two dimensions. As shown in Table 19, the younger students spent more time on social media than the older students.

Table 19

Amount of Time Spent on Social Media by Age

\begin{tabular}{|c|c|c|c|c|c|c|}
\hline \multirow[t]{2}{*}{ Age groups } & \multicolumn{6}{|c|}{ Frequency and percent of respondents } \\
\hline & OHOURS $>$ & 1-2 HOURS & 5-6 HOURS & 7-10 HOURS & 11 HOURS OR MORE & TOTAL \\
\hline $\begin{array}{l}\text { Q89: } 18-20 \\
\text { (A) }\end{array}$ & $\begin{array}{r}0.76 \% \\
2\end{array}$ & $\begin{array}{r}14.89 \% \\
39 \\
\mathrm{BC}\end{array}$ & $\begin{array}{r}39.31 \% \\
103\end{array}$ & $\begin{array}{r}32.82 \% \\
86\end{array}$ & $\begin{array}{r}12.21 \% \\
32 \\
\text { BC }\end{array}$ & $\begin{array}{r}63.29 \% \\
262\end{array}$ \\
\hline $\begin{array}{l}\text { Q89: 21-25 } \\
\text { (B) }\end{array}$ & $\begin{array}{r}2.73 \% \\
3\end{array}$ & $\begin{array}{r}37.27 \% \\
41 \\
\text { AC }\end{array}$ & $\begin{array}{r}19.09 \% \\
21\end{array}$ & $\begin{array}{r}40.00 \% \\
44\end{array}$ & $\begin{array}{r}0.91 \% \\
1 \\
\mathrm{~A}\end{array}$ & $\begin{array}{r}26.57 \% \\
110\end{array}$ \\
\hline $\begin{array}{l}\text { Q89: } 26 \text { and } \\
\text { above (C) }\end{array}$ & $\begin{array}{r}0.00 \% \\
0\end{array}$ & $\begin{array}{r}100.00 \% \\
42 \\
A B\end{array}$ & $\begin{array}{r}0.00 \% \\
0\end{array}$ & $\begin{array}{r}0.00 \% \\
0\end{array}$ & $\begin{array}{r}0.00 \% \\
0 \\
\mathrm{~A}\end{array}$ & $\begin{array}{r}10.14 \% \\
42\end{array}$ \\
\hline $\begin{array}{l}\text { Total } \\
\text { Respondents }\end{array}$ & 5 & 122 & 124 & 130 & 33 & 414 \\
\hline
\end{tabular}

Notes. ${ }^{*} \mathrm{p}=0.05,95 \%$ confidence.

With regard to their self-perception of addictive behavior, $79 \%$ of the students admitted that they were spending too much time on the Internet, and $65 \%$ believed that they were spending too much time on social media (Figures 4-5). Furthermore, about 70\% admitted that they were addicted to the Internet, and 57\% thought they were addicted to social media (Figures 6-7). As shown in Tables 20-23, there was no significant gender difference for these variables.

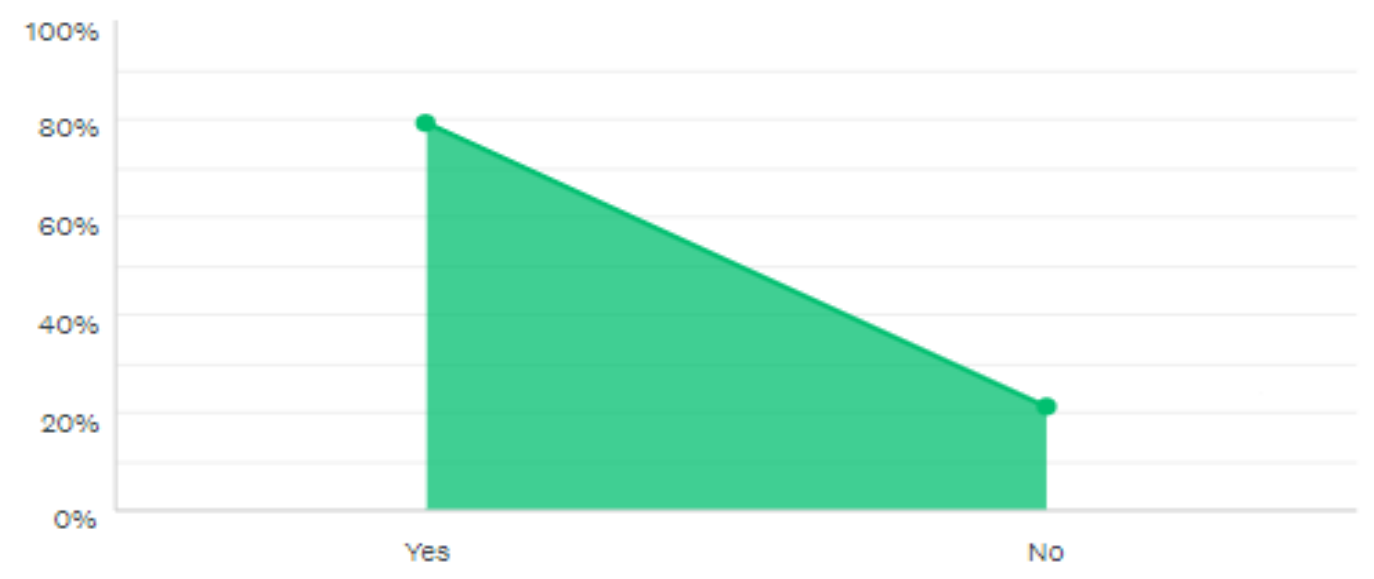

Figure 4. Spending too much time on the Internet. 


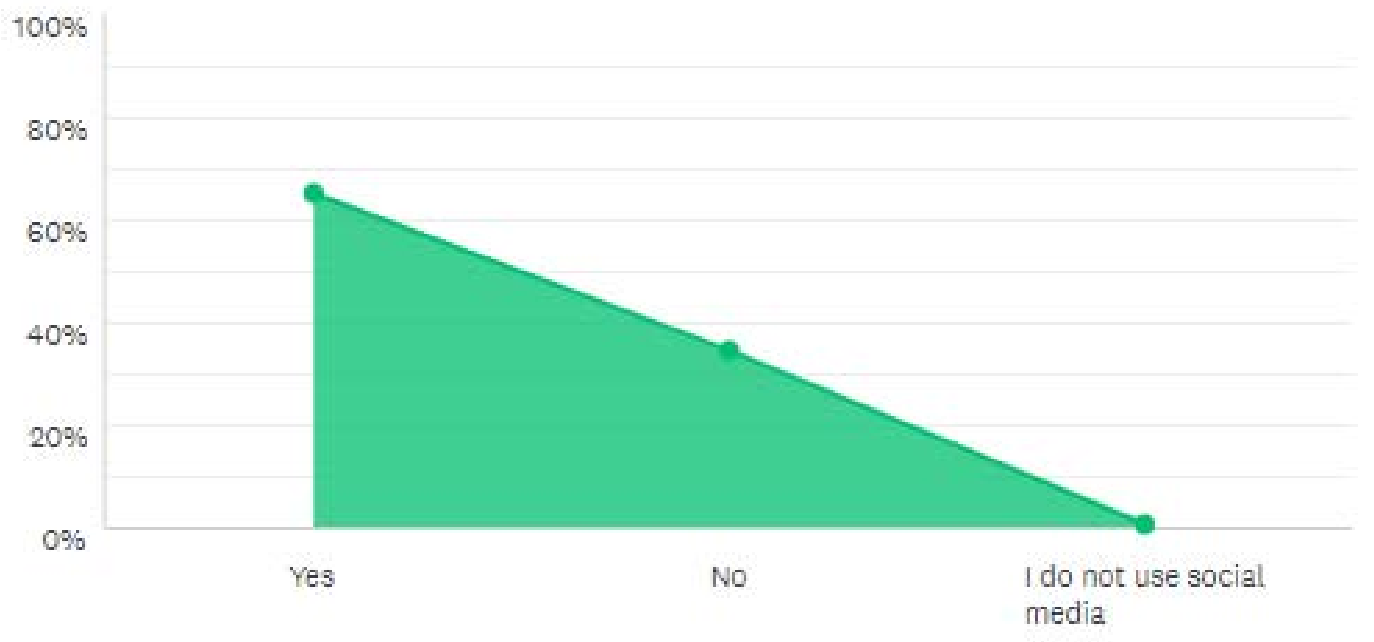

Figure 5. Spending too much time on social media.

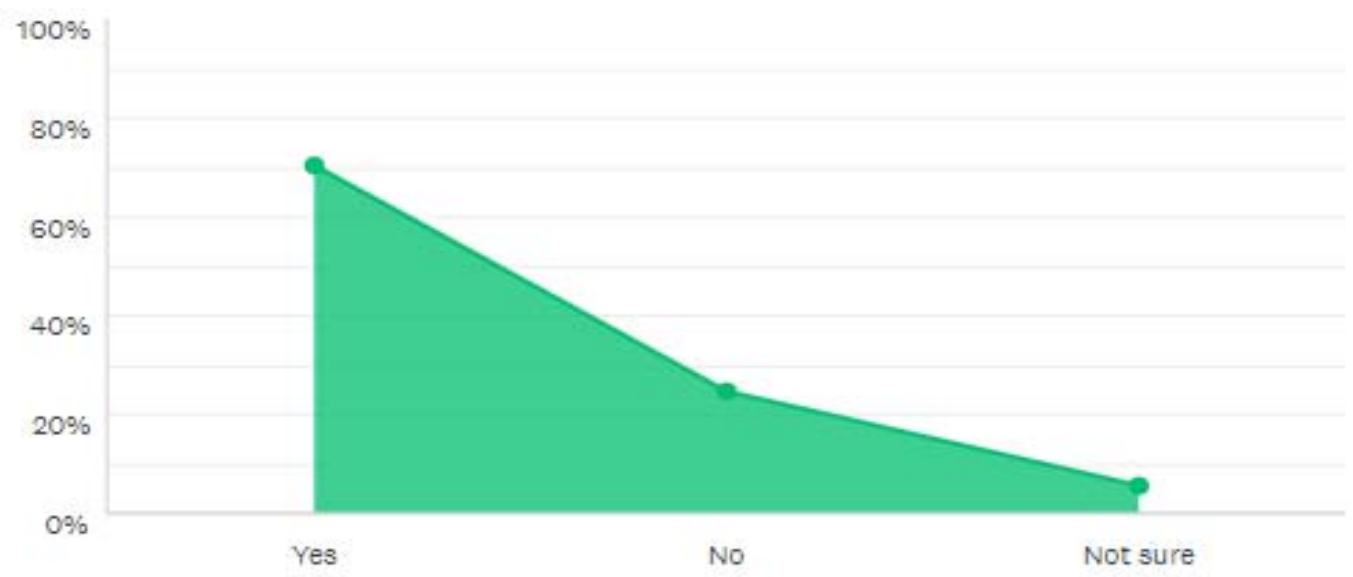

Figure 6. Addicted to the Internet.

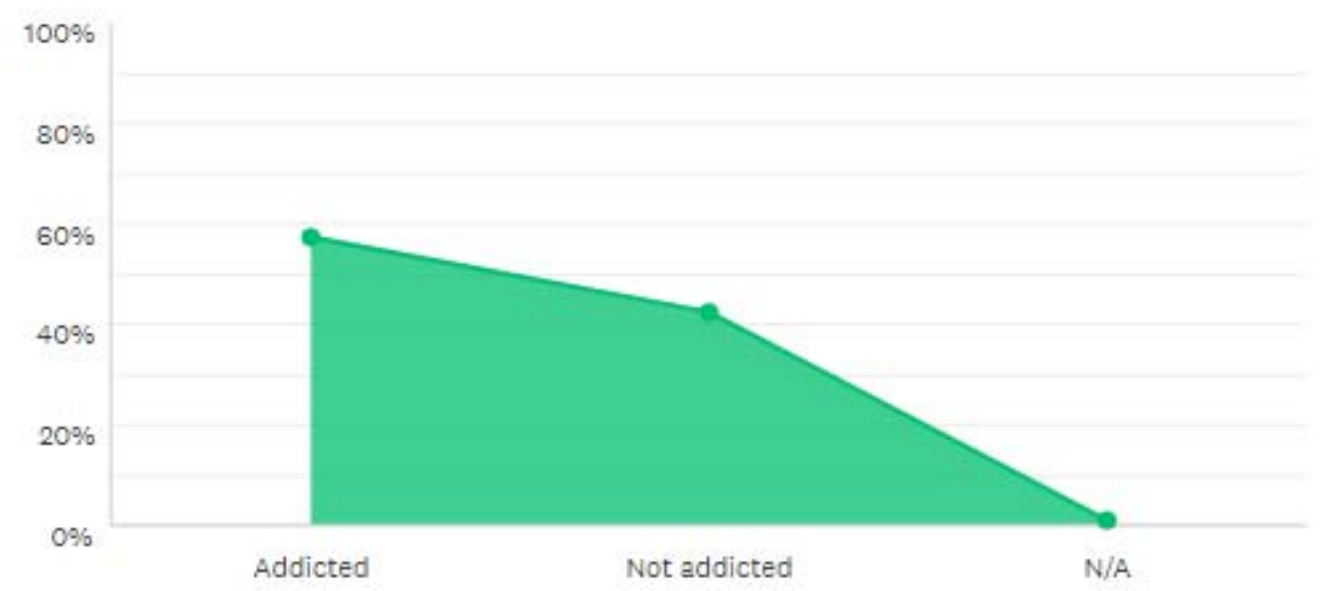

Figure 7. Addicted to social media. 
Table 20

Spending Too Much Time Spent on Internet by Gender

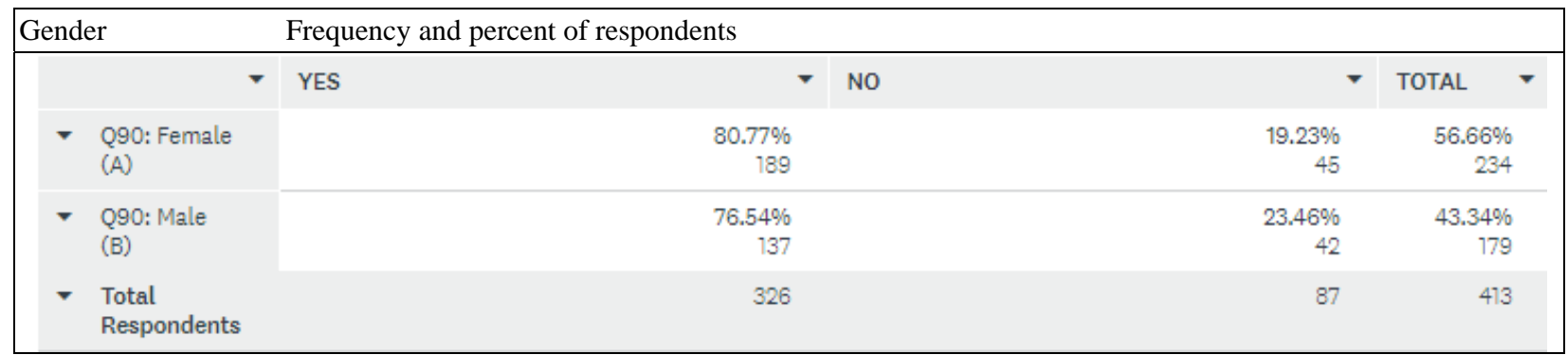

Table 21

Spending Too Much on Social Media by Gender

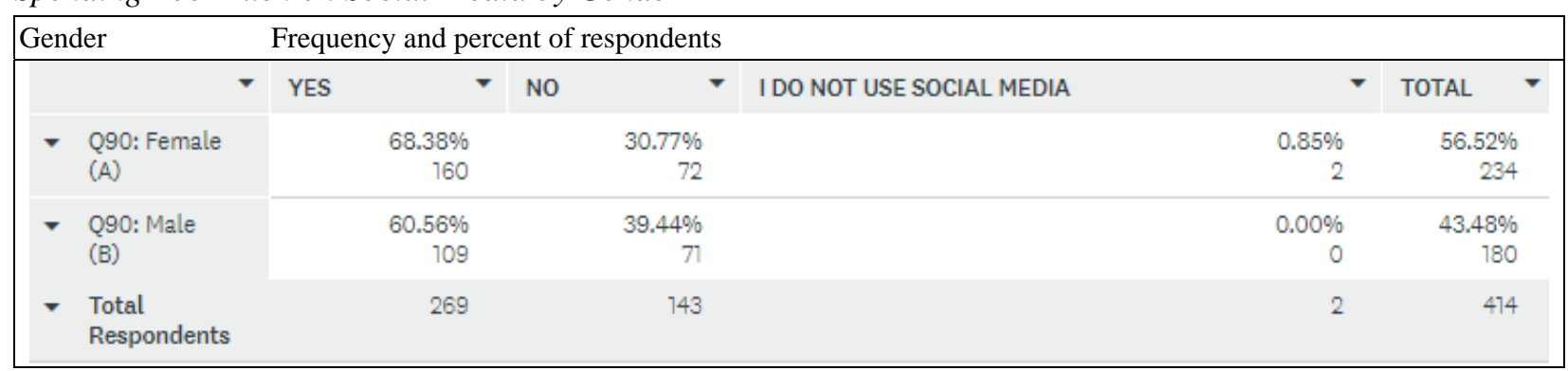

Table 22

Perceived Addiction to Internet by Gender

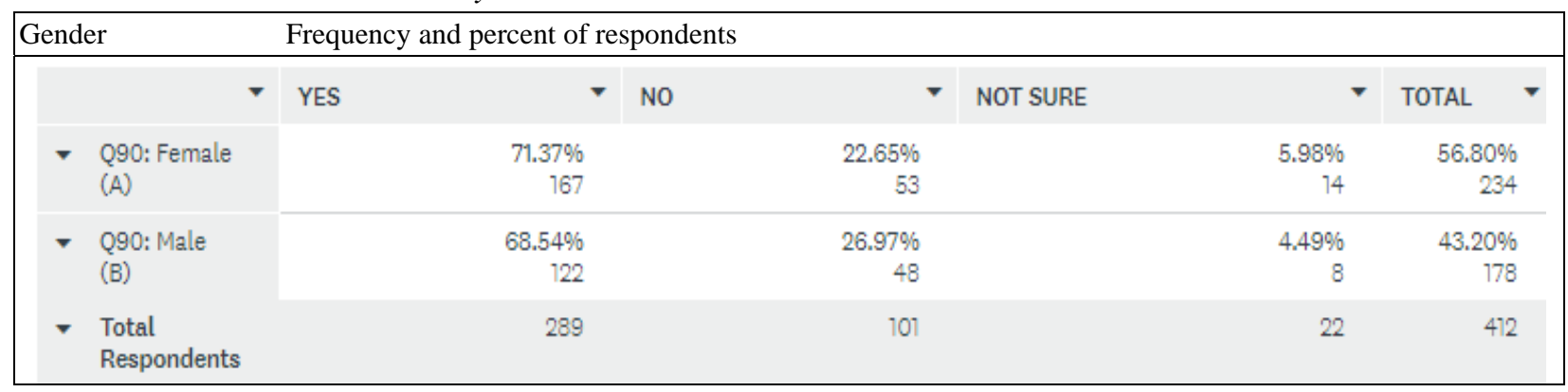

Table 23

Perceived Addiction to Social Media by Gender

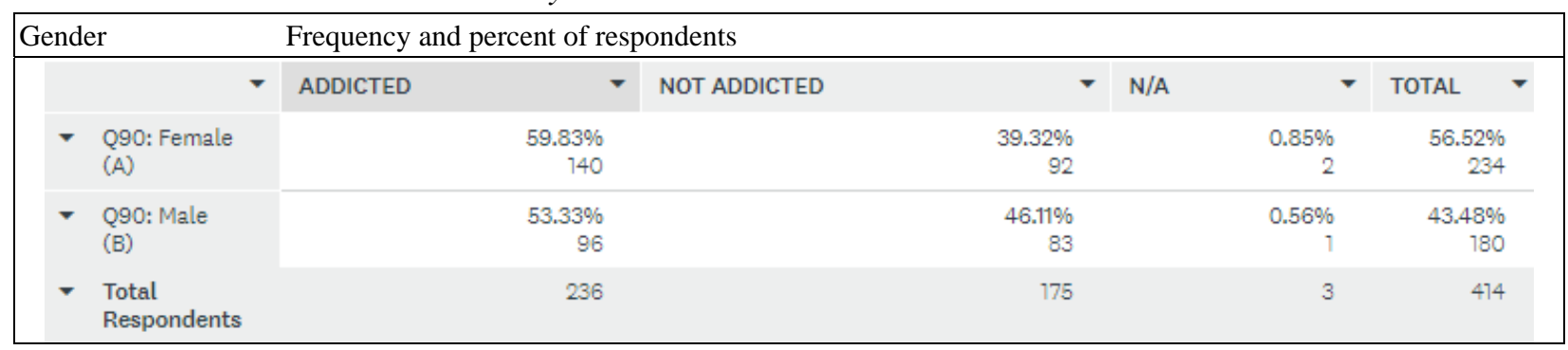

In terms of age variation, a larger percentage of younger students admitted that they were spending too much time on the Internet and social media, and that they were addicted to the Internet and social media (Tables 24-27). 
Table 24

Spending Too Much Time on Internet by Age

\begin{tabular}{|c|c|c|c|}
\hline \multirow[t]{2}{*}{ Age groups } & \multicolumn{3}{|l|}{ Frequency and percent of respondents } \\
\hline & YES & NO & TOTAL \\
\hline $\begin{array}{l}\text { Q89: 18-20 } \\
\text { (A) }\end{array}$ & $\begin{array}{r}82.76 \% \\
216 \\
\mathrm{C}\end{array}$ & $\begin{array}{r}17.24 \% \\
45 \\
\mathrm{C}\end{array}$ & $\begin{array}{r}63.20 \% \\
261\end{array}$ \\
\hline $\begin{array}{l}\text { Q89: } 21-25 \\
\text { (B) }\end{array}$ & $\begin{array}{r}86.36 \% \\
95 \\
\mathrm{C}\end{array}$ & $\begin{array}{r}13.64 \% \\
15 \\
\mathrm{C}\end{array}$ & $\begin{array}{r}26.63 \% \\
110\end{array}$ \\
\hline $\begin{array}{l}\text { Q89: } 26 \text { and } \\
\text { above (C) }\end{array}$ & $\begin{array}{r}35.71 \% \\
15 \\
A B\end{array}$ & $\begin{array}{r}64.29 \% \\
27 \\
A B\end{array}$ & $\begin{array}{r}10.17 \% \\
42\end{array}$ \\
\hline $\begin{array}{l}\text { Total } \\
\text { Respondents }\end{array}$ & 326 & 87 & 413 \\
\hline
\end{tabular}

Notes. ${ }^{*} \mathrm{p}=0.05,95 \%$ confidence.

Table 25

Spending Too Much Time on Social Media by Age

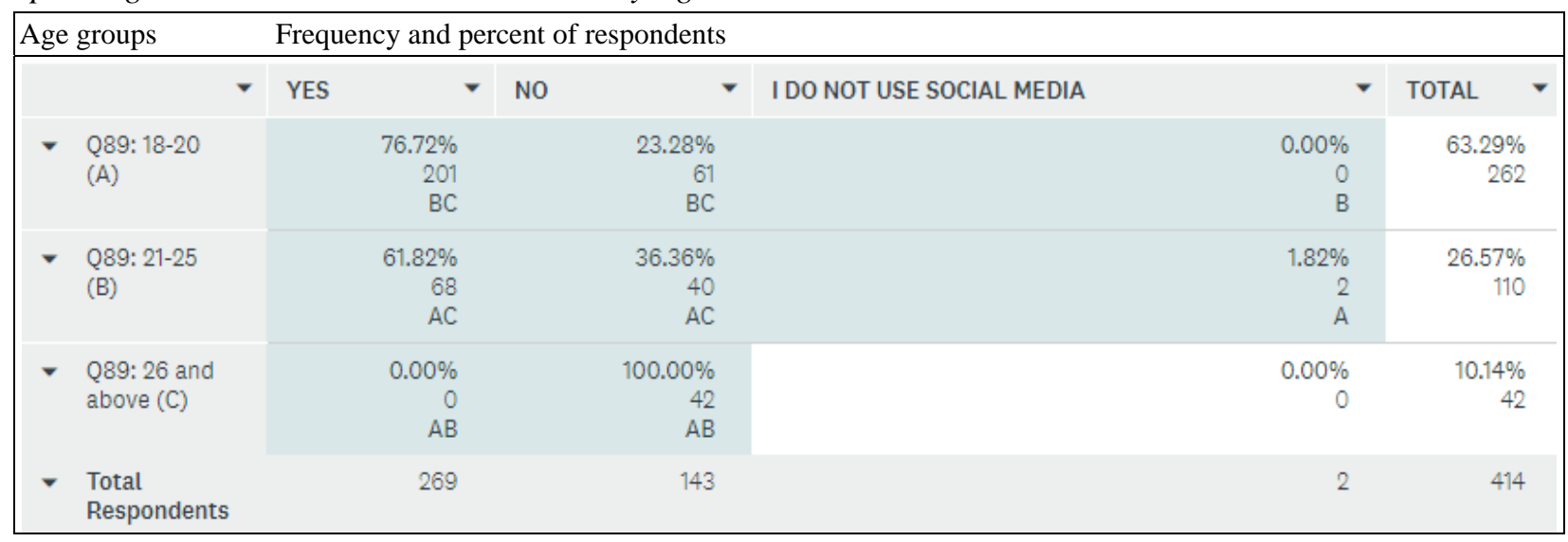

Notes. ${ }^{*} \mathrm{p}=0.05,95 \%$ confidence.

Table 26

Perceived Addiction to Internet by Age

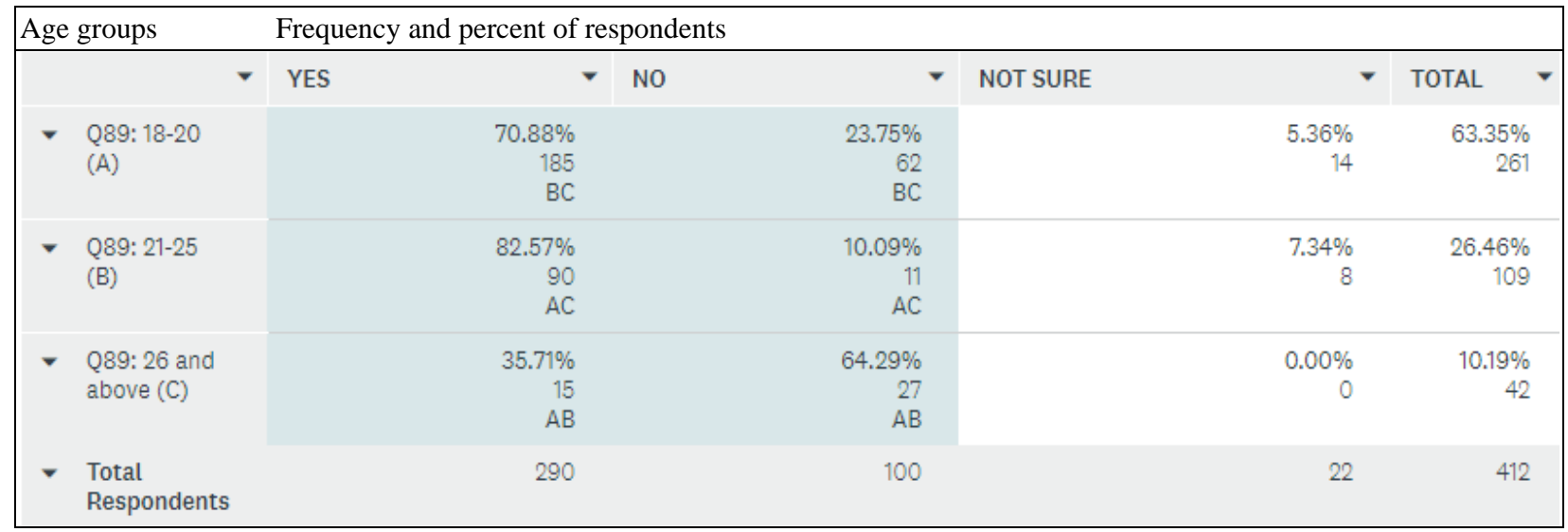

Notes. ${ }^{*} \mathrm{p}=0.05,95 \%$ confidence. 
Table 27

Perceived Addiction to Social Media by Age

\begin{tabular}{|c|c|c|c|c|c|c|c|}
\hline \multicolumn{2}{|c|}{ Age groups } & \multicolumn{6}{|c|}{ Frequency and percent of respondents } \\
\hline & $\checkmark$ & ADDICTED & NOT ADDICTED & 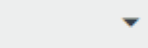 & N/A & $\checkmark$ & TOTAL \\
\hline & $\begin{array}{l}\text { Q89: } 18-20 \\
\text { (A) }\end{array}$ & $\begin{array}{r}67.18 \% \\
176 \\
\text { BC }\end{array}$ & & $\begin{array}{r}32.44 \% \\
85 \\
\mathrm{C}\end{array}$ & & $\begin{array}{r}0.38 \% \\
1\end{array}$ & $\begin{array}{r}63.29 \% \\
262\end{array}$ \\
\hline & $\begin{array}{l}\text { Q89: } 21-25 \\
\text { (B) }\end{array}$ & $\begin{array}{r}55.45 \% \\
61 \\
\text { AC }\end{array}$ & & $\begin{array}{r}42.73 \% \\
47 \\
C\end{array}$ & & $\begin{array}{r}1.82 \% \\
2\end{array}$ & $\begin{array}{r}26.57 \% \\
110\end{array}$ \\
\hline & $\begin{array}{l}\text { Q89: } 26 \text { and } \\
\text { above (C) }\end{array}$ & $\begin{array}{r}0.00 \% \\
0 \\
A B\end{array}$ & & $\begin{array}{r}100.00 \% \\
42 \\
A B\end{array}$ & & $\begin{array}{r}0.00 \% \\
0\end{array}$ & $\begin{array}{r}10.14 \% \\
42\end{array}$ \\
\hline$v$ & $\begin{array}{l}\text { Total } \\
\text { Respondents }\end{array}$ & 237 & & 174 & & 3 & 414 \\
\hline
\end{tabular}

Notes. ${ }^{\mathrm{p}} \mathrm{p}=0.05,95 \%$ confidence.

When asked what would their life be like without the social media, 59\% admitted that they would be bored, $38 \%$ would be depressed, $77 \%$ would be out of touch, and $57 \%$ would be lonely (Table 28). Furthermore, in the absence of the social media, $80 \%$ believed that they would be more productive and would communicate more face-to-face, $73 \%$ would do more exercise, $64 \%$ would play more sports, and $76 \%$ would read more books (Table 28). As shown in Table 29, there was no significant gender difference for most of the dimensions.

Table 28

Life Without Social Media

\begin{tabular}{|lcc|}
\hline Feelings and activities & Frequency and percent of respondents \\
\hline - I would be bored & $58.74 \%$ & 242 \\
\hline - I would be depressed & $38.11 \%$ & 157 \\
\hline - I would be out of touch & $61.41 \%$ & 253 \\
\hline - I would be lonely & $41.50 \%$ \\
\hline - I would communicate more face-to-face & $80.34 \%$ & 331 \\
\hline - I would do more exercise & $79.61 \%$ & 328 \\
\hline - I would play more sports & $72.57 \%$ \\
\hline - I would read more books & $63.83 \%$ & 299 \\
\hline - It would not make any difference as I do not use social media & $75.49 \%$ & 263 \\
\hline Total Respondents: 412 & $5.58 \%$ & 311 \\
\hline
\end{tabular}


Table 29

Life Without Social Media by Gender

\begin{tabular}{|c|c|c|c|c|c|c|c|c|c|c|}
\hline \multirow[t]{2}{*}{ Gender } & \multicolumn{10}{|c|}{ Frequency and percent of respondents } \\
\hline & $\begin{array}{l}\text { I } \\
\text { WOULD, } \\
\text { BE } \\
\text { BORED }\end{array}$ & $\begin{array}{l}\text { I WOULD BE } \\
\text { DEPRESSED }\end{array}$ & $\begin{array}{l}\text { I } \\
\text { WOULD } \\
\text { BE OUT - } \\
\text { OF } \\
\text { TOUCH }\end{array}$ & $\begin{array}{l}\text { I } \\
\text { WOULD } \\
\text { BE } \\
\text { LONELY }\end{array}$ & $\begin{array}{l}\text { IWOULD BE } \\
\text { MORE } \\
\text { PRODUCTIVE }\end{array}$ & $\begin{array}{l}\text { IWOULD } \\
\text { COMMUNICATE } \\
\text { MORE FACE- } \\
\text { TO-FACE }\end{array}$ & $\begin{array}{l}\text { I WOULD } \\
\text { DO MORE } \\
\text { EXERCISE }\end{array}$ & $\begin{array}{l}\text { I } \\
\text { WOULD } \\
\text { PLAY } \\
\text { MORE } \\
\text { SPORTS }\end{array}$ & $\begin{array}{l}\text { I } \\
\text { WOULD } \\
\text { READ - } \\
\text { MORE } \\
\text { BOOKS }\end{array}$ & $\begin{array}{l}\text { IT WOULD } \\
\text { NOT MAKE } \\
\text { ANY } \\
\text { DIFFERENCE } \\
\text { AS I DO NOT } \\
\text { USE SOCIAL } \\
\text { MEDIA }\end{array}$ \\
\hline $\begin{array}{l}\text { Q90: Female } \\
\text { (A) }\end{array}$ & $\begin{array}{r}61.97 \% \\
145\end{array}$ & $\begin{array}{r}38.46 \% \\
90\end{array}$ & $\begin{array}{r}66.24 \% \\
155\end{array}$ & $\begin{array}{r}44.02 \% \\
103\end{array}$ & $\begin{array}{r}82.48 \% \\
193\end{array}$ & $\begin{array}{r}79.06 \% \\
185\end{array}$ & $\begin{array}{r}71.37 \% \\
167\end{array}$ & $\begin{array}{r}58.55 \% \\
137\end{array}$ & $\begin{array}{r}79.91 \% \\
187\end{array}$ & $\begin{array}{r}4.27 \% \\
10\end{array}$ \\
\hline $\begin{array}{l}\text { Q90: Male } \\
\text { (B) }\end{array}$ & $\begin{array}{r}54.24 \% \\
96\end{array}$ & $\begin{array}{r}37.29 \% \\
66\end{array}$ & $\begin{array}{r}54.80 \% \\
97\end{array}$ & $\begin{array}{r}37.85 \% \\
67\end{array}$ & $\begin{array}{r}77.40 \% \\
137\end{array}$ & $\begin{array}{r}80.23 \% \\
142\end{array}$ & $\begin{array}{r}74.01 \% \\
131\end{array}$ & $\begin{array}{r}70.62 \% \\
125\end{array}$ & $\begin{array}{r}69.49 \% \\
123\end{array}$ & $\begin{array}{r}7.34 \% \\
13\end{array}$ \\
\hline $\begin{array}{l}\text { Total } \\
\text { Respondents }\end{array}$ & 241 & 156 & 252 & 170 & 330 & 327 & 298 & 262 & 310 & 23 \\
\hline & OTHER & PECIFY) & & & & & & & & \\
\hline
\end{tabular}

In terms of age variation, the data revealed that younger students expressed more concerns about their life without social media as compared to older students (Table 30).

Table 30

Life Without Social Media by Age

\begin{tabular}{|c|c|c|c|c|c|c|c|c|c|c|}
\hline \multirow[t]{2}{*}{ Age groups } & \multicolumn{10}{|c|}{ Frequency and percent of respondents } \\
\hline & $\begin{array}{l}\text { I } \\
\text { WOULD } \\
\text { BE } \\
\text { BORED }\end{array}$ & $\begin{array}{l}\text { I WOULD BE } \\
\text { DEPRESSED }\end{array}$ & $\begin{array}{l}\text { I } \\
\text { WOULD } \\
\text { BE OUT - } \\
\text { OF } \\
\text { TOUCH }\end{array}$ & $\begin{array}{l}\text { I } \\
\text { WOULD } \\
\text { BE } \\
\text { LONELY }\end{array}$ & $\begin{array}{l}\text { I WOULD BE } \\
\text { MORE } \\
\text { PRODUCTIVE }\end{array}$ & $\begin{array}{l}\text { I WOULD } \\
\text { COMMUNICATE } \\
\text { MORE FACE- } \\
\text { TO-FACE }\end{array}$ & $\begin{array}{l}\text { I WOULD } \\
\text { DO MORE } \\
\text { EXERCISE }\end{array}$ & $\begin{array}{l}\text { I } \\
\text { WOULD } \\
\text { PLAY - } \\
\text { MORE } \\
\text { SPORTS }\end{array}$ & $\begin{array}{l}\text { I } \\
\text { WOULD } \\
\text { READ - } \\
\text { MORE } \\
\text { BOOKS }\end{array}$ & $\begin{array}{l}\text { IT WOULD } \\
\text { NOT MAKE } \\
\text { ANY } \\
\text { DIFFERENCE - } \\
\text { AS I DO NOT } \\
\text { USE SOCIAL } \\
\text { MEDIA }\end{array}$ \\
\hline $\begin{array}{l}\text { Q89: } 18-20 \\
\text { (A) }\end{array}$ & $\begin{array}{r}70.11 \% \\
183 \\
\mathrm{C}\end{array}$ & $\begin{array}{r}43.30 \% \\
113 \\
C\end{array}$ & $\begin{array}{r}75.10 \% \\
196 \\
\text { BC }\end{array}$ & $\begin{array}{r}47.89 \% \\
125 \\
C\end{array}$ & $\begin{array}{r}85.06 \% \\
222\end{array}$ & $\begin{array}{r}81.61 \% \\
213\end{array}$ & $\begin{array}{r}71.65 \% \\
187 \\
C\end{array}$ & $\begin{array}{r}60.92 \% \\
159 \\
\mathrm{C}\end{array}$ & $\begin{array}{r}76.25 \% \\
199 \\
C\end{array}$ & $\begin{array}{r}1.15 \% \\
3 \\
C\end{array}$ \\
\hline $\begin{array}{l}\text { Q89: 21-25 } \\
\text { (B) }\end{array}$ & $\begin{array}{r}54.63 \% \\
59 \\
\mathrm{C}\end{array}$ & $\begin{array}{r}39.81 \% \\
43 \\
\mathrm{C}\end{array}$ & $\begin{array}{r}52.78 \% \\
57 \\
\text { AC }\end{array}$ & $\begin{array}{r}42.59 \% \\
46 \\
C\end{array}$ & $\begin{array}{r}87.04 \% \\
94\end{array}$ & $\begin{array}{r}83.33 \% \\
90\end{array}$ & $\begin{array}{r}80.56 \% \\
87\end{array}$ & $\begin{array}{r}73.15 \% \\
79 \\
\text { C }\end{array}$ & $\begin{array}{r}80.56 \% \\
87\end{array}$ & $\begin{array}{r}2.78 \% \\
3 \\
C\end{array}$ \\
\hline $\begin{array}{l}\text { Q89: } 26 \text { and } \\
\text { above (C) }\end{array}$ & $\begin{array}{r}0.00 \% \\
0 \\
A B\end{array}$ & $\begin{array}{r}0.00 \% \\
0 \\
A B\end{array}$ & $\begin{array}{r}0.00 \% \\
0 \\
A B\end{array}$ & $\begin{array}{r}0.00 \% \\
0 \\
A B\end{array}$ & $\begin{array}{r}35.71 \% \\
15\end{array}$ & $\begin{array}{r}59.52 \% \\
25\end{array}$ & $\begin{array}{r}59.52 \% \\
25 \\
\mathrm{~A}\end{array}$ & $\begin{array}{r}59.52 \% \\
25 \\
A B\end{array}$ & $\begin{array}{r}59.52 \% \\
25 \\
\mathrm{~A}\end{array}$ & $\begin{array}{r}40.48 \% \\
17 \\
A B\end{array}$ \\
\hline $\begin{array}{l}\text { Total } \\
\text { Respondents }\end{array}$ & 242 & 156 & 253 & 171 & 331 & 328 & 299 & 263 & 311 & 23 \\
\hline
\end{tabular}

Notes. ${ }^{*} \mathrm{p}=0.05,95 \%$ confidence.

When asked what would their life be like without the Internet in general, about $75 \%$ admitted that they would be bored, $54 \%$ would be depressed, $61 \%$ would be out of touch, and $42 \%$ would be lonely (Table 31 ). Furthermore, in the absence of the Internet, $80 \%$ believed that they would be more productive and would communicate more face-to-face, $75 \%$ would read more books, $74 \%$ would do more exercise, and $63 \%$ would play more sports (Table 31). 
Table 31

Life Without Internet by

\begin{tabular}{|lc|}
\hline Feelings and activities & Frequency and percent of respondents \\
\hline I would be bored & 310 \\
\hline I would be depressed & $74.88 \%$ \\
\hline I would be out of touch & $54.11 \%$ \\
\hline I would be lonely & $77.29 \%$ \\
\hline I would be more productive & 524 \\
\hline I would do more exercise & $37.00 \%$ \\
\hline I would play more sports & 320 \\
\hline I would read more books & 323 \\
\hline Total Respondents: 414 & $30.02 \%$ \\
\hline
\end{tabular}

In terms of gender variation, no significant difference was found for most of the dimensions (Table 32). However, in terms of age variation, data revealed that younger students expressed more concerns about their life without the Internet as compared to older students (Table 33).

Table 32

Life Without Internet by Gender

\begin{tabular}{|c|c|c|c|c|c|c|c|c|c|}
\hline \multicolumn{10}{|c|}{ Feelings and activities Frequency and percent of respondents } \\
\hline $\overrightarrow{ }$ & $\begin{array}{l}\text { I } \\
\text { WOULD } \\
\text { BE } \\
\text { BORED }\end{array}$ & $\begin{array}{l}\text { I WOULD BE } \\
\text { DEPRESSED }\end{array}$ & $\begin{array}{l}\text { I } \\
\text { WOULD } \\
\text { BE OUT - } \\
\text { OF } \\
\text { TOUCH }\end{array}$ & $\begin{array}{l}\text { I } \\
\text { WOULD } \\
\text { BE } \\
\text { LONELY }\end{array}$ & $\begin{array}{l}\text { I WOULD BE } \\
\text { MORE } \\
\text { PRODUCTIVE }\end{array}$ & $\begin{array}{l}\text { I WOULD } \\
\text { COMMUNICATE, } \\
\text { MORE FACE- } \\
\text { TO-FACE }\end{array}$ & $\begin{array}{l}\text { I WOULD } \\
\text { DO MORE } \\
\text { EXERCISE }\end{array}$ & $\begin{array}{l}\text { I } \\
\text { WOULD } \\
\text { PLAY } \\
\text { MORE } \\
\text { SPORTS }\end{array}$ & $\begin{array}{l}\text { I } \\
\text { WOULD } \\
\text { READ } \\
\text { MORE } \\
\text { BOOKS }\end{array}$ \\
\hline $\begin{array}{l}\text { Q90: Female } \\
\text { (A) }\end{array}$ & $\begin{array}{r}76.39 \% \\
178\end{array}$ & $\begin{array}{r}54.51 \% \\
127\end{array}$ & $\begin{array}{r}80.26 \% \\
187\end{array}$ & $\begin{array}{r}57.51 \% \\
134\end{array}$ & $\begin{array}{r}80.69 \% \\
188\end{array}$ & $\begin{array}{r}79.40 \% \\
185\end{array}$ & $\begin{array}{r}72.10 \% \\
168\end{array}$ & $\begin{array}{r}59.23 \% \\
138\end{array}$ & $\begin{array}{r}75.97 \% \\
177\end{array}$ \\
\hline $\begin{array}{l}\text { Q90: Male } \\
\text { (B) }\end{array}$ & $\begin{array}{r}72.78 \% \\
131\end{array}$ & $\begin{array}{r}53.33 \% \\
96\end{array}$ & $\begin{array}{r}73.33 \% \\
132\end{array}$ & $\begin{array}{r}56.11 \% \\
101\end{array}$ & $\begin{array}{r}74.44 \% \\
134\end{array}$ & $\begin{array}{r}82.22 \% \\
148\end{array}$ & $\begin{array}{r}75.56 \% \\
136\end{array}$ & $\begin{array}{r}66.67 \% \\
120\end{array}$ & $\begin{array}{r}62.22 \% \\
112\end{array}$ \\
\hline $\begin{array}{l}\text { Total } \\
\text { Respondents }\end{array}$ & 309 & 223 & 319 & 235 & 322 & 333 & 304 & 258 & 289 \\
\hline
\end{tabular}


Table 33

Life Without Internet by Age

\begin{tabular}{|c|c|c|c|c|c|c|c|c|c|}
\hline \multirow[t]{2}{*}{ Age groups } & \multicolumn{9}{|c|}{ Frequency and percent of respondents } \\
\hline & $\begin{array}{l}\text { I } \\
\text { WOULD } \\
\text { BE } \\
\text { BORED }\end{array}$ & $\begin{array}{l}\text { I WOULD BE } \\
\text { DEPRESSED }\end{array}$ & $\begin{array}{l}\text { I } \\
\text { WOULD } \\
\text { BE OUT } \\
\text { OF } \\
\text { TOUCH }\end{array}$ & $\begin{array}{l}\text { I } \\
\text { WOULD } \\
\text { BE } \\
\text { LONELY }\end{array}$ & $\begin{array}{l}\text { I WOULD BE } \\
\text { MORE } \\
\text { PRODUCTIVE }\end{array}$ & $\begin{array}{l}\text { I WOULD } \\
\text { COMMUNICATE } \\
\text { MORE FACE- } \\
\text { TO-FACE }\end{array}$ & $\begin{array}{l}\text { I WOULD } \\
\text { DO MORE } \\
\text { EXERCISE }\end{array}$ & $\begin{array}{l}\text { I } \\
\text { WOULD } \\
\text { PLAY } \\
\text { MORE } \\
\text { SPORTS }\end{array}$ & $\begin{array}{l}\text { I } \\
\text { WOULD } \\
\text { READ } \\
\text { MORE } \\
\text { BOOKS }\end{array}$ \\
\hline $\begin{array}{l}\text { Q89: } 18-20 \\
\text { (A) }\end{array}$ & $\begin{array}{r}78.93 \% \\
206 \\
\mathrm{C}\end{array}$ & $\begin{array}{r}50.96 \% \\
133\end{array}$ & $\begin{array}{r}80.84 \% \\
211 \\
C\end{array}$ & $\begin{array}{r}54.41 \% \\
142\end{array}$ & $\begin{array}{r}74.71 \% \\
195\end{array}$ & $\begin{array}{r}77.78 \% \\
203\end{array}$ & $\begin{array}{r}69.35 \% \\
181 \\
\mathrm{C}\end{array}$ & $\begin{array}{r}57.47 \% \\
150 \\
\mathrm{C}\end{array}$ & $\begin{array}{r}66.67 \% \\
174 \\
\mathrm{C}\end{array}$ \\
\hline $\begin{array}{l}\text { Q89: 21-25 } \\
\text { (B) }\end{array}$ & $\begin{array}{r}80.91 \% \\
89 \\
\mathrm{C}\end{array}$ & $\begin{array}{r}69.09 \% \\
76 \\
C\end{array}$ & $\begin{array}{r}85.45 \% \\
94 \\
C\end{array}$ & $\begin{array}{r}71.82 \% \\
79 \\
\mathrm{C}\end{array}$ & $\begin{array}{r}78.18 \% \\
86\end{array}$ & $\begin{array}{r}80.00 \% \\
88\end{array}$ & $\begin{array}{r}74.55 \% \\
82\end{array}$ & $\begin{array}{r}60.91 \% \\
67 \\
C\end{array}$ & $\begin{array}{r}66.36 \% \\
73 \\
C\end{array}$ \\
\hline $\begin{array}{l}\text { Q89: } 26 \text { and } \\
\text { above (C) }\end{array}$ & $\begin{array}{r}35.71 \% \\
15 \\
A B\end{array}$ & $\begin{array}{r}35.71 \% \\
15 \\
\mathrm{~B}\end{array}$ & $\begin{array}{r}35.71 \% \\
15 \\
A B\end{array}$ & $\begin{array}{r}35.71 \% \\
15 \\
\mathrm{~B}\end{array}$ & $\begin{array}{r}100.00 \% \\
42\end{array}$ & $\begin{array}{r}100.00 \% \\
42\end{array}$ & $\begin{array}{r}100.00 \% \\
42 \\
\mathrm{~A}\end{array}$ & $\begin{array}{r}100.00 \% \\
42 \\
A B\end{array}$ & $\begin{array}{r}100.00 \% \\
42 \\
A B\end{array}$ \\
\hline $\begin{array}{l}\text { Total } \\
\text { Respondents }\end{array}$ & 310 & 224 & 320 & 236 & 323 & 333 & 305 & 259 & 289 \\
\hline
\end{tabular}

Notes. ${ }^{*} \mathrm{p}=0.05,95 \%$ confidence.

\section{Discussion and Conclusions}

This study examined the smartphone and social media addiction among Generation Z.

The results revealed that almost all Gen Zers (99\%) own a smartphone, and 98\% of them use their smartphones to connect to the Internet most of the time. More than half of Gen Zers spend nine hours or more in a typical day using their smartphones, and nearly half of them spend 3-8 hours per day on their smartphones. The data did not show any significant difference in terms of gender or age groups, which indicates that both males and females, and younger as well as older students are spending a significant amount of time on their smartphones on a daily basis.

The results also showed that nearly half of Gen Zers use mediated interpersonal communication or digital communication (via smartphone, social media, email, texting, instant messaging, etc.) most often than face-to-face communication. Moreover, about three-fifths admitted that the use of social media had decreased their face-to-face communication. Both males and females used mediated interpersonal communication more often than face-to-face communication. However, younger students used mediated interpersonal communication more often as compared to older students.

In terms of the nature of smartphone usage, one notable finding reveals that more than two-thirds of Gen Zers use their smartphones for doing research for class assignments. Other usage of smartphones included making phone calls, text messaging, emailing, checking the news, posting or viewing photos and videos on social media, watching TV and movies online, playing music and games, purchasing online, accessing dating sites, and searching and applying for jobs. With regard to the use of social media, the results revealed that Snapchat, Instagram, Facebook, and Twitter are the most widely used social media among Generation Z with majority of them having multiple social media accounts. In terms of frequency of usage, more than half of Gen Zers accessed various social media several times a day, while nearly 1 in 5 admitted accessing social media every hour of the day. In terms of gender variation, data showed that more female students accessed the social media every hour of the day compared to males. As for age variation, the findings revealed that the younger 
students accessed the social media more often than the older students. With regard to the amount of time devoted to social media, nearly one-third of the students admitted using social media for 7-10 hours per day, and slightly less than one-third of the students spent 5-6 hours per day on social media. There were no significant differences in terms of gender for most of the dimensions. However, a greater percentage of younger students spent more time on social media than older students.

As for Generation Z's perception of their addiction to smartphone and social media, 4 in 5 students admitted that they were spending an excessive amount of time on the Internet, and two-thirds believed that they were spending too much time on social media. Furthermore, 7 in 10 students admitted that they were addicted to the Internet, and nearly two-thirds believed they were addicted to social media. Although there was no significant gender difference, younger students were more likely to admit that they were spending too much time on the Internet and social media, and that they were addicted to the Internet and social media.

When asked what would their life be like without the social media, roughly 6 in 10 students admitted that they would be bored and lonely, 4 in 10 would be depressed, and 8 in 10 would be out of touch. Furthermore, in the absence of the Internet, about 8 in 10 believed that they would be more productive and would communicate more face-to-face, 7 in 10 would read more books and would do more exercise, and 6 in 10 would play more sports. Younger students expressed more concerns about their life without the Internet and social media as compared to older students. There was no significant gender difference for this variable.

The aforementioned discussion of data analysis indicates that although there are some positive uses, it appears that Generation Zers are spending an excessive amount of time on their smartphones and social media, and that most of them are addicted to their smartphones and social media. These findings may have significant implications for counseling and intervention for parents, educators, and policy makers. Furthermore, the results may also have significance for marketers, advertisers, mass media, and apps creators who target the Gen $\mathrm{Z}$ with their products, services, and contents, as majority of Gen Zers use their smartphones and social media for networking, online purchasing, research, news consumption, and entertainment.

This research was based on a non-random sample and focused on Generation Z, and thereby limiting the ability to generalize the results to the larger population. Future research with a random sample could focus on additional variables and dimensions relating to addictive usage of smartphone and social media, and its implications for the personal, educational, and professional lives of all demographics.

\section{References}

Al-Menayes, J. (2016). The fear of missing out scale: Validation of the Arabic version and correlation with social media addiction. International Journal of Applied Psychology, 6, 41-46.

Andrew, H. (June 5, 2018). Snapchat publishes new insights into Generation Z. Social Media Today.

Bian, M., \& Leung, L. (2014). Linking loneliness, shyness, smartphone addiction symptoms, and patterns of smartphone use to social capital. Social Science Computer Review, 33(1), 61-79.

Billieux, J., Maurage, P., Lopez-Fernandez, O., Kuss, D. J., \& Griffiths, M. D. (2015). Can disordered mobile phone use be considered a behavioral addiction? An update on current evidence and a comprehensive model for future research. Current Addiction Report, 2, 156-162.

Buglass, S. L., Binder, J. F., Betts, L. R., \& Underwood, J. D. M. (2017). Motivators of online vulnerability: The impact of social network site use and FOMO. Computers in Human Behavior, 66, 248-255.

Bragazzi, N. L., \& Del Puente, G. (2014). A proposal for including nomophobia in the new DSMV. Psychology Research and Behavior Management, 7, 155-160. 
Caplan, S. E. (2003). Preference for online social interaction: A theory of problematic Internet use and psychosocial well-being. Communication Research, 30, 625-648.

Chen, Y., Liang, C., Mai, X., \& Zhong, Q. (2016). General deficit in inhibitory control of excessive smartphone users: Evidence from an event-related potential study. Frontiers in Psychology, 7, 511.

Christofides, E., Muise, A., \& Desmarais, S. (2012). Risky disclosures on Facebook: The effect of having a bad experience on online behavior. Journal of Adolescent Research, 27(6), 714-731.

Hawi, N., \& Samaha, M. (2016). The relations among social media addiction, self-esteem, and life satisfaction in university students. Social Science Computer Review, 35(5), 576-586.

Hong, F., \& Chiu, D. (2012). A model of the relationship between psychological characteristics, mobile phone addiction and use of mobile phones by Taiwanese university female students. Computer Human Behavior, 28, 2152-2159.

Jeong, S., Kim, H., Yum, J., \& Hwang, Y. (2016). What type of content are smartphone users addicted to?: SNS vs. games. Computers in Human Behavior, 54, 10-17.

King, D., Delfabbro, P., \& Griffiths, M. (2010). The convergence of gambling and digital media: Implications for gambling in young people. Journal of Gambling Studies, 26, 175-187.

Kross, E., Verduyn, P., Demiralp, E., Park, J., Lee, D. S., \& Lin, N. (2013). Facebook use predicts declines in subjective well-being in young adults. PLOS One, 8(8), e69841.

Kuss, D. J., \& Griffiths, M. D. (2011). Online social networking and addiction-a review of the psychological literature. International Journal of Environmental Research. Public Health, 8, 3528-3552.

Kuss, D. J., \& Griffiths, M. D. (2012). Internet gaming addiction: A systematic review of empirical research. International Journal of Mental Health Addiction, 10, 278-296.

Lee, J., Sung, M., Song, S., Lee, Y., Lee, J., Cho, S., Park, M., \& Shin, Y. (2016). Psychological factors associated with smartphone addiction in South Korean adolescents. The Journal of Early Adolescence, 38(3), 288-302.

Lin, Y. C., Lee, P. H., Lin, S. H., Lin, L. R., \& Chang, T. B. (2015). Time distortion associated with smartphone addiction: Identifying smartphone addiction via a mobile application. Journal of Psychiatric Research, 65, 139-145.

Lopez-Fernandez, O., Kuss, D. J., Griffiths, M. D., \& Billieux, J. (2015). The conceptualization and assessment of problematic mobile phone use. In Encyclopedia of mobile phone behavior (pp. 591-606). Hershey, Pa: IGI Global.

Megan, A. W., William, M. B., \& Eric, L. (2016). The digital self: How social media serves as a setting that shapes youth's emotional experiences. Adolescent Research Review, 1(2), 163-173.

Miller, L., \& Lu, W. (2018, August 20). Gen Z is set to outnumber millennials. Bloomberg Economic.

Montag, P. W. (2016). Carpe diem instead of losing your social mind: Beyond digital addiction and why we all suffer from digital overuse. Cogent Psychology, 3(1), 1157281.

Moreno, M. A., Jelenchick, L. A., Egan, K. G., Cox, E., Young, H., Gannon, K. E., \& Becker, T. (2011). Feeling bad on Facebook: Depression disclosures by college students on a social networking site. Depression and Anxiety, 28(6), 447-455.

Müller, K. W., Dreier, M., Duven, E., Giralt, S., Beutel, M. E., \& Wölfling, K. A. (2016). Hidden type of Internet addiction? Intense and addictive use of social networking sites in adolescents. Computers in Human Behavior, 55, 172-177.

Muise, A., Christofides, E., \& Desmarais, S. (2009). More information than you ever wanted: Does Facebook bring out the green-eyed monster of jealousy? Cyberpsychology \& Behavior, 12(4), 441-444.

Nazir, S., \& Samaha, M. (2016). Relationships among smartphone addiction, anxiety, and family relations. Behavior \& Information Technology, 36(10), 1046-1052.

Oberst, U., Wegmann, E., Stodt, B., Brand, M., \& Chamarro, A. (2017). Negative consequences from heavy social networking in adolescents: The mediating role of fear of missing out. Journal of Adolescence, 55, 51-60.

Przybylski, A. K., Murayama, K., DeHaan, C. R., \& Gladwell, V. (2013). Motivational, emotional, and behavioral correlates of fear of missing out. Computers in Human Behavior, 29, 1841-1848.

Seemiller, C., \& Grace, M. (2016). Generation Z goes to college. San Francisco, California: Jossey-Brass.

Schimmenti, A., Passanisi, A., Gervasi, A. M., Manzella, S., \& Fama, F. I. (2015). Insecure attachment attitudes in the onset of problematic Internet use among late adolescents. Child Psychiatry Human Development, 45, 588-595.

Schou, A. C., \& Pallesen, S. (2014). Social network site addiction—an overview. Current Pharmaceutical Design, 20(25), 4053-4061.

Smith, A., \& Monica, A. (2016). Social media use in 2016. Pew Research Center Internet \& Technology. Pew Research Center.

Song, I., LaRose, R., Eastin, M. S., \& Lin, C. A. (2004). Internet gratifications and internet addiction: On the uses and abuses of new media. Cyberpsychology and Behavior, 7, 384-394. 
Statista Facts on Social Networks. (2015). Average numbers of hours per day spent by social media users on all social media channels. Retrieved from https://www.statista.com/statistics/270229/usage-duration-of-social networks-by-country/

Statista Facts on Social Networks. (2016). Retrieved from https://www.statista.com/topics/1164/social-networks/

Tsitsika, A. K., Tzavela, E. C., Janikian, M., Ólafsson, K., Iordache, A., Schoenmakers, T. M., Tzavara, C., \& Richardson, C. (2014). Online social networking in adolescence: Patterns of use in six European countries and links with psychosocial functioning. Journal of Adolescent Health, 55, 141-147.

Wang, C. W., Ho, R. T. H., Chan, C. L. W., \& Tse, S. (2015). Exploring personality characteristics of Chinese adolescents with internet-related addictive behaviors: Trait differences for gaming addiction and social networking addiction. Addictive Behavior, 42, 32-35.

Wood, S. (2013). Generation Z as consumers: Trends and innovation. Institute for Emerging Issues. N.C. State University.

Wu, A. M. S., Cheung, V. I., Ku, L., \& Hung, E. P. W. (2013). Psychological risk factors of addiction to social networking sites among Chinese smartphone users. Journal of Behavioral Addictions, 2(3), 160-166. 